\title{
Moduli Spaces of Commutative Ring Spectra
}

\author{
P. G. Goerss and M. J. Hopkins*
}

\begin{abstract}
Let $E$ be a homotopy commutative ring spectrum, and suppose the ring of cooperations $E_{*} E$ is flat over $E_{*}$. We wish to address the following question: given a commutative $E_{*}$-algebra $A$ in $E_{*} E$-comodules, is there an $E_{\infty}$-ring spectrum $X$ with $E_{*} X \cong A$ as comodule algebras? We will formulate this as a moduli problem, and give a way - suggested by work of Dwyer, Kan, and Stover - of dissecting the resulting moduli space as a tower with layers governed by appropriate André-Quillen cohomology groups. A special case is $A=E_{*} E$ itself. The final section applies this to discuss the Lubin-Tate or Morava spectra $E_{n}$.
\end{abstract}

Some years ago, Alan Robinson developed an obstruction theory based on Hochschild cohomology to decide whether or not a homotopy associative ring spectrum actually has the homotopy type of an $A_{\infty}$-ring spectrum. In his original paper on the subject [35] he used this technique to show that the Morava $K$-theory spectra $K(n)$ can be realized as an $A_{\infty}$-ring spectrum; subsequently, in [3], Andrew Baker used these techniques to show that a completed version of the Johnson-Wilson spectrum $E(n)$ can also be given such a structure. Then, in the mid-90s, the second author and Haynes Miller showed that the entire theory of universal deformations of finite height formal group laws over fields of non-zero characteristic can be lifted to $A_{\infty}$-ring spectra in an essentially unique way. This implied, in particular, that the Morava $E$-theory (or Lubin-Tate) spectra $E_{n}$ were $A_{\infty}$ (which could have been deduced from Baker's work), but it also showed much more. Indeed, the theory of Lubin and Tate [25] gives a functor from a category of finite height formal group laws to the category of complete local rings, and one way to state the results of [34] is that this functor factors in an essentially unique way through $A_{\infty}$-ring spectra. It was the solution of the diagram lifting problem that gave this result its additional heft; for example, it implied that the Morava stabilizer group acted on $E_{n}-$ simply because Lubin-Tate theory implied that this group acted on $\left(E_{n}\right)_{*}$.

In this paper, we would like to carry this program several steps further. One step forward would be to address $E_{\infty}$-ring spectra rather than $A_{\infty}$-ring spectra. There is an existing literature on this topic developed by Robinson and others, some based on $\Gamma$-homology. See [36], [37], and [4]. This can be used, to prove, among other things, that the spectra $E_{n}$ are $E_{\infty}$, and we guess that the obstruction theory we uncover here reduces to that theory. Another

${ }^{*}$ The authors were partially supported by the National Science Foundation. 
step forward would be to write down and try to solve the realization problem as a moduli problem: what is the space of all possible realizations of a spectrum as an $A_{\infty}$ or $E_{\infty}$-ring spectrum, and how can one calculate the homotopy type of this space? Robinson's original work on Morava $K$-theory implied that this space would often have many components or, put another way, that there could be many $A_{\infty}$-realizations of a fixed homotopy associative spectrum. A third step forward would be to build a theory that easily globalizes; that is, we might try to realize a diagram of commutative rings by a diagram of $E_{\infty}$-ring spectra, or we might try to come to terms with some sheaf of commutative rings. One particular such sheaf we have in mind is the structure sheaf of a moduli stack of elliptic curves, but one could also consider the structure sheaf of the moduli stack of formal group laws. In fact, many of our examples arise by examining pieces of this latter stack. A final step forward would be to build a theory that passes directly from algebra to $E_{\infty}$ or $A_{\infty}$-ring spectra, rather than by an intermediate pass through the stable homotopy category. This would be in line with the Lubin-Tate lifting of the previous paragraph.

Let us expand on some of these points.

The $E_{\infty}$-realization problem is more subtle than the $A_{\infty}$-problem. If $X$ is a spectrum, the free $A_{\infty}$-ring spectrum $\mathcal{A}(X)$ on $X$ has the homotopy type of $\vee_{n \geq 0} X^{\wedge n}$, so that if $E_{*}$ is a homology theory with a Künneth spectral sequence and $E_{*} X$ is flat over $E_{*}$, then $E_{*} \mathcal{A}(X)$ is isomorphic to the tensor algebra over $E_{*}$ on $E_{*}(X)$. This basic computation underlies much of the rest of the theory. However, the free $E_{\infty}$-ring spectrum $\mathcal{E}(X)$ has the homotopy type of

$$
\bigvee_{n \geq 0}\left(E \Sigma_{n}\right)_{+} \wedge_{\Sigma_{n}} X^{\wedge n}
$$

where $E \Sigma_{n}$ is a free contractible $\Sigma_{n}$-space. To compute $E_{*} \mathcal{E}(X)$ would require, at the very least, knowledge of $E_{*} B \Sigma_{n}$ and, practically, one would need define and understand a great deal about the $E_{*}$ Dyer-Lashof algebra. Even if this calculation could be made, one would be left with a another problem. In trying to realize some commutative $E_{*}$-algebra $A$ in $E_{*} E$ comodules as an $E_{\infty}$ ring spectrum, one might not be able or might not want to stipulate a Dyer-Lashof algebra structure on $A$. Indeed, our problem is simply to realize $A$ as a commutative algebra - not to realize $A$ with some stipulated Dyer-Lashof algebra structure. Thus, any theory we build must allow for this flexibility.

Our solution is to resolve an $E_{\infty}$ operad by a simplicial operad which at once yields this desired flexibility and the possibility of computing the $E_{*}$-homology of a free object. This has the drawback, of course, of getting us involved with the cohomology of simplicial objects over simplicial operads. Part of the point of this paper is to demonstrate that this is workable and, in fact, leads into familiar territory. See section 6 and, more generally, [18]. It is here that the Dyer-Lashof operations - which have to arise somewhere - reappear in an explicit manner.

The moduli space of all possible realizations of a commutative $E_{*}$-algebra $A$ in $E_{*} E$-comodules is a Dwyer-Kan classification space in the sense of [12]. Let $\mathcal{E}(A)$ be the category whose objects are $E_{\infty}$-spectra $X$ with $E_{*} X \cong A$ as 
commutative $E_{*}$-algebras in $E_{*} E$-comodules. The morphisms are morphisms of $E_{\infty}$-ring spectra which are $E_{*}$-isomorphisms. The moduli space $\mathcal{T} \mathcal{M}(A)$ of all realizations of $A$ is the nerve of this category. According to Dwyer and Kan, there is a weak equivalence

$$
\mathcal{T} \mathcal{M}(A) \simeq \coprod_{X} B \operatorname{Aut}(X)
$$

where $X$ runs over $E_{*}$-isomorphism classes of objects in $\mathcal{E}(A)$ and $\operatorname{Aut}(X)$ is the monoid of self equivalences of a cofibrant-fibrant model for $X$. Pleasant as this result is, it is not really a computation in this setting; for example, we cannot immediately tell if this space is non-empty or not. Thus, we need some sort of decomposition of $\mathcal{T} \mathcal{M}(A)$ with computable and, ideally, algebraic input. This is accomplished in Section 5; the algebraic input is an André-Quillen cohomology of $A$ with coefficients in shifted versions of $A$. The basic theory for this kind of construction is spelled out in [6]; the exact result we obtain is gotten by combining Proposition 5.2, Proposition 5.5, and Theorem 5.8. Keeping track of basepoints in the resulting tower decomposition of $\mathcal{T} \mathcal{M}(A)$ yields an obstruction theory for realizing $A$. The details are in 5.9.

This material works equally well for $A_{\infty}$-structures. In this case the AndréQuillen cohomology we obtain is exactly the Quillen cohomology of associative algebras; see [30]. Except possibly in degree zero, this is a shift of Hochschild homology, as one might expect from Robinson's work.

One detail about this theory is worth examining here: the moduli space $\mathcal{T} \mathcal{M}(A)$ and its decomposition do not require the existence of a homotopy associative or commutative ring spectrum $X$ with $E_{*} X \cong A$. Of course, in practice, such an $X$ might be required for another reason. For example, in the basic case where $A=E_{*} E$, then we need $X=E$ to exist and be a homotopy commutative ring spectrum.

Here is an outline of the paper. In the first section, we confront the foundations. There are many competing, but Quillen equivalent, models for spectra in the literature. We write down exactly what we need from any given model, and point out that there exist models which have the requisite properties. The next two sections are about resolutions, first of operads, and then of spectra and algebras in spectra over operads. Here is where the resolution (or " $E_{2}$ ") model category structures of Dwyer, Kan, and Stover ([14],[15]) come in. We use an elegant formulation of this theory due to Bousfield [9]. Section 4 is devoted to a definition of the requisite André-Quillen cohomology groups and to a spectral sequence for computing the homotopy type of the mapping space of $E_{\infty}$-maps between two $E_{\infty}$-ring spectra. Again we emphasize that the $E_{2}$-term of this spectral sequence requires no knowledge of a Dyer-Lashof structure. Section 5 introduces the decomposition of the moduli space. Section 6 talks about methods of calculation, and Section 7 applies these techniques to the example of the diagram of Lubin-Tate spectra - the Hopkins-Miller theorem in $E_{\infty}$-ring spectra. The result is the same as for $A_{\infty}$-case.

Two notation conventions: First, for two objects in a model category, the space of maps $\operatorname{map}(X, Y)$ will always mean the derived simplicial mapping 
set of maps. All our model categories will be simplicial model categories; hence $\operatorname{map}(X, Y)$ is weakly equivalence to the simplicial mapping set out of cofibrant model for $X$ into a fibrant model for $Y$. Alternatively, one can write down $\operatorname{map}(X, Y)$ as the nerve of an appropriate diagram category, such as the DwyerKan hammock localization [13].

Second, if $X$ is a simplicial object in some category $\mathcal{C}$, then we will say $X$ is $s$-free if the underlying degeneracy diagram is free. This means there are objects $Z_{n} \in \mathcal{C}$ and isomorphisms

$$
X_{n} \cong \coprod_{\phi:[n] \rightarrow[m]} Z_{m}
$$

where $\phi$ runs over the surjections in the ordinal number category. Furthermore, these isomorphisms respect degeneracy maps of $X$ in the obvious way.

\section{Contents}

1 The ground category: which category of spectra to use?

2 Simplicial spectra over simplicial operads

3 Resolutions

4 André-Quillen cohomology

5 The moduli space of realizations

6 Computing with $E_{\infty}$ operads.

\section{The ground category: which category of spec- tra to use?}

In the original drafts of these notes, and in other papers on this subject, we used the category of spectra developed by Lewis, May, and Steinberger in [26]. This had a number advantages for us; in particular, every object is fibrant in this category, and the role of the operads is explicit, even elegant. We needed every object to be fibrant so that we could apply the theory of Stover resolutions and the $E_{2}$-model categories of [14] to build our resolutions. However, since that time Bousfield [9] and Jardine [24] have both shown that it is possible to remove the condition that every object is fibrant and still have a theory of $E_{2}$-model categories - or, as we (and Bousfield) prefer to call them, resolution model categories. This opened up the possibility of using any one of a number of other models for spectra - in fact almost any will now do. We get a nice synergy 
with operads if the underlying category has a closed symmetric monoidal smash product, so we will choose one of the current such models with this property. The point of this section is to produce an exact statement of what we need, along with some examples. This statement is broken into two parts: see Axioms 1.1 and Axioms 1.4 below.

We note that we are surrendering one facet of the previous discussion by this move away from LMS spectra. It turns out the homotopy category of $C$-algebras in spectra, where $C$ is some operad, depends only on the weak equivalence type of $C$ in the naïvest possible sense, which is in sharp distinction to the usual results about, say, spaces. (The exact result is below, in Theorem 1.6.) For the LMS spectra this fact comes down to the fact that one must use operads over the linear isometries operad, and such operads always have free actions by the symmetric groups. For the categories under discussion here, however, the reasons are less transparent, because they are buried in the definition of the smash product - and only an avatar of this freeness appears in the last of our axioms (in 1.4) for spectra.

To begin, here is exactly we will need about the symmetric monoidal structure. For the language of model categories, see [21]. In particular, the concepts of a monoidal model category and of a module over a monoidal category is discussed in Chapter 4.2 of that work. Specifically, simplicial sets are a monoidal model category and a simplicial model category is a module category over simplicial sets. For any category of spectra, the action of a simplicial set $K$ on a spectrum $X$ should be, up to weak equivalence, given by the formula

$$
X \otimes K=X \wedge K_{+}
$$

whenever this makes homotopical sense. Here the functor $(-)_{+}$means adjoin a disjoint basepoint. This is the point of axiom 3 below.

1.1 Axioms for Spectra. We will assume that we have some category $\mathcal{S}$ of spectra which satisfy the following conditions:

1.) The category $\mathcal{S}$ is a cofibrantly generated proper stable simplicial model category Quillen equivalent to the Bousfield-Friedlander [10] category of simplicial spectra; furthermore, $\mathcal{S}$ has a generating set of cofibrations and a generating set of acyclic cofibrations with cofibrant source.

2.) The category $\mathcal{S}$ has a closed symmetric monoidal smash product which descends to the usual smash product on the homotopy category; furthermore, with that monoidal structure, $\mathcal{S}$ is a monoidal model category.

3.) The smash product behaves well with respect to the simplicial structure; specifically, if $S$ is the unit object of the smash product, then there is a natural monoidal isomorphism

$$
X \otimes K \stackrel{\cong}{\longrightarrow} X \wedge(S \otimes K) .
$$


Note that Axiom 1 guarantees, among other things, that the homotopy category is the usual stable category.

We immediately point out that at least three of the favorite candidates for such a category satisfy these axioms. Symmetric spectra built from simplicial sets are discussed in [22]; symmetric spectra and orthogonal spectra built using topological spaces are defined and discussed in [27]. The spectra known as $S$ modules are built from topological spaces and are discussed in [16]. It is worth pointing out that $S$-modules are built on and depend on LMS spectra [26]. The categories of symmetric spectra and of orthogonal spectra have at least two Quillen equivalent model category structures on them. For the next result either would do; later results will require the "positive" model category structure of [27], §14.

1.2 Theorem. The category of symmetric spectra (in spaces or simplicial sets), the category of orthogonal spectra, and the category of S-modules satisfy the axioms 1.1.

Proof. Axioms 1, 2, and 3 are explicit in [22] for symmetric spectra in simplicial sets. For othogonal spectra, symmetric spectra in spaces, and $S$-modules, we note that these categories are not immediately simplicial model categories, but topological model categories. But any topological model category is automatically a simplicial model category via the realization functor. Then Axioms 1,2 , and 3 are in [27] for symmetric and orthogonal spectra and in [16] for $S$-modules.

As with all categories modeling the stable homotopy category one has to explicitly spell out what one means by some familiar terms.

1.3 Notation for Spectra. The following remarks and notation will be used throughout this paper.

1.) We will use the words cofibrant and cellular interchangeably. The generating cofibrations of $\mathcal{S}$ are usually some sort of inclusion of spheres into cells.

2.) We will write $[X, Y]$ for the morphisms in the homotopy category $\mathbf{H o}(\mathcal{S})$. As usual, this is $\pi_{0}$ for some derived space of maps. See point (5) below.

3.) In the category $\mathcal{S}$ it is possible (indeed usual) that the unit object $S$ for the smash product ("the zero-sphere") is not cofibrant. We will write $S^{k}$, $-\infty<k<\infty$ for a cofibrant model for the $k$-sphere unless we explicitly state otherwise. In this language the suspension functor on the homotopy category is induced by

$$
X \mapsto X \wedge S^{1} .
$$

Also the suspension spectrum functor from pointed simplicial sets to spectra is, by axiom 3 , modeled by

$$
K \mapsto S^{0} \wedge K \stackrel{\text { def }}{=} \frac{S^{0} \otimes K}{S^{0} \otimes *}
$$


Note that if the unit object $S$ is not cofibrant, the functor $S \otimes(-)$ is not part of a Quillen pair.

4.) Let $K$ be a simplicial set and $X \in \mathcal{S}$. We may write $X \wedge K_{+}$for the tensor object $X \otimes K$. This is permissible by axiom 3 and in line with the geometry. The exponential object in $\mathcal{S}$ will be written $X^{K}$.

5.) We will write $\operatorname{map}(X, Y)$ or $\operatorname{map}_{\mathcal{S}}(X, Y)$ for the derived simplicial set of maps between two objects of $\mathcal{S}$. Thus, $\operatorname{map}(X, Y)$ is the simplicial mapping space between some fibrant-cofibrant models ("bifibrant") models for $X$ and $Y$. This can be done functorially if necessary, as the category $\mathcal{S}$ is cofibrantly generated. Alternatively, we could use some categorical construction, such as the Dwyer-Kan hammock localization. Note that with this convention

$$
\pi_{0} \operatorname{map}(X, Y)=[X, Y] .
$$

6.) We will write $F(X, Y)$ for the function spectrum of two objects $X, Y \in \mathcal{S}$. The closure statement in Axiom 2 of 1.1 amounts to the statement that

$$
\operatorname{Hom}_{\mathcal{S}}(X, F(Y, Z)) \cong \operatorname{Hom}_{\mathcal{S}}(X \wedge Y, Z) .
$$

This can be derived:

$$
\operatorname{map}(X, R F(Y, Z)) \simeq \operatorname{map}\left(X \wedge^{L} Y, Z\right)
$$

where the $R$ and $L$ refer to the total derived functors and $\operatorname{map}(-,-)$ is the derived mapping space. In particular

$$
\pi_{k} R F(Y, Z) \cong\left[\Sigma^{k} Y, Z\right] .
$$

7.) If $X$ is cofibrant and $Y$ is fibrant, then there is a natural weak equivalence

$$
\operatorname{map}(X, Y) \simeq \operatorname{map}\left(S^{0}, F(X, Y)\right)
$$

and the functor $\operatorname{map}\left(S^{0},-\right)$ is the total right derived functor of the suspension spectrum functor from pointed simplicial sets to $\mathcal{S}$. Thus we could write

$$
\operatorname{map}(X, Y) \simeq \Omega^{\infty} F(X, Y) .
$$

In particular, $\operatorname{map}(X, Y)$ is canonically weakly equivalent to an infinite loop space.

We need a notation for iterated smash products. So, define

$$
X^{(n)} \stackrel{\text { def }}{=} \underset{\longleftarrow}{X \wedge} \stackrel{\cdots \wedge X}{\longrightarrow} .
$$

This paper is particularly concerned with the existence of $A_{\infty}$ and $E_{\infty}$-ring spectrum structures. Thus we we must introduce the study of operads acting on spectra. 
Let $\mathcal{O}$ denote the category of operads in simplicial sets. Our major source of results for this category is [33]. The category $\mathcal{O}$ is a cofibrantly generated simplicial model category where $C \rightarrow D$ is a weak equivalence or fibration if each of the maps $C(n) \rightarrow D(n)$ is a weak equivalence or fibration of $\Sigma_{n}$-spaces in the sense of equivariant homotopy theory. Thus, for each subgroup $H \subseteq \Sigma_{n}$, the induced map $C(n)^{H} \rightarrow D(n)^{H}$ is a weak equivalence or fibration. The existence of the model category structure follows from the fact that the forgetful functor from operads to the category with objects $X=\{X(n)\}_{n \geq 0}$ with each $X(n)$ a $\Sigma_{n}$-space has a left adjoint with enough good properties that the usual lifting lemmas apply.

If $C$ is an operad in simplicial sets, then we have a category of $\operatorname{Alg}_{C}$ of algebras over $C$ is spectra. These are exactly the algebras over the triple

$$
X \mapsto C(X) \stackrel{\text { def }}{=} \vee_{n \geq 0} C(n) \otimes_{\Sigma_{n}} X^{(n)} .
$$

Note that we should really write $X^{(n)} \otimes_{\Sigma_{n}} C(n)$, but we don't.

The object $C(*) \cong S \otimes C(0)$ is the initial object of $\operatorname{Alg}_{C}$. If the operad is reduced - that is, $C(0)$ is a point - then this is simply $S$ itself.

If $f: C \rightarrow D$ is morphism of operads, then there is a restriction of structure functor $f_{*}: \operatorname{Alg}_{D} \rightarrow \operatorname{Alg}_{C}$, and this has a left adjoint

$$
f^{*} \stackrel{\text { def }}{=} D \otimes_{C}(-): \operatorname{Alg}_{C} \rightarrow \operatorname{Alg}_{D}
$$

The categories $\mathrm{Alg}_{C}$ are simplicial categories in the sense of Quillen and both the restriction of structure functor and its adjoint are continuous. Indeed, if $X \in \operatorname{Alg}_{C}$ and $K$ is a simplicial set, and if $X^{K}$ is the exponential object of $K$ in $\mathcal{S}$, then $X^{K}$ is naturally an object in $\operatorname{Alg}_{C}$ and with this structure, it is the exponential object in $\mathcal{S}^{C}$. Succinctly, we say the forgetful functor makes exponential objects. It also makes limits and reflexive coequalizers, filtered colimits, and geometric realization of simplicial objects.

Here is our second set of axioms. The numbering continues that of Axioms 1.1.

1.4 Axioms for Spectra. Suppose we are given some category $\mathcal{S}$ of spectra satisfying the axioms of 1.1. Then we further require that

4.) For a fixed operad $C \in \mathcal{O}$, define a morphism of $X \rightarrow Y$ of $C$-algebras in spectra to be a weak equivalence or fibration if it is so in spectra. Then the category $\mathrm{Alg}_{C}$ becomes a cofibrantly generated simplicial model category.

5.) Let $n \geq 1$ and let $K \rightarrow L$ be a morphism of $\Sigma_{n}$ spaces which is a weak equivalence on the underlying spaces. Then for all cofibrant spectra $X$, the induced map on orbit spectra

$$
K \otimes_{\Sigma_{n}} X^{(n)} \rightarrow L \otimes_{\Sigma_{n}} X^{(n)}
$$

is a weak equivalence of spectra. 
We immediately note that we have examples.

1.5 Proposition. Let $\mathcal{S}$ be any of the categories of symmetric spectra in topological spaces, orthogonal spectra, or $S$-modules. Then $\mathcal{S}$ satisfies the axioms of 1.4.

Proof. First, axiom 4. For $S$-modules, this is nearly obvious, from a standard argument that goes back to Quillen, but see also [33] or [5] for the argument in the context of operads. In brief, since $\mathrm{Alg}_{C}$ has a functorial path object and the forgetful functor to $\mathcal{S}$ creates filtered colimits in $\mathrm{Alg}_{C}$, we need only supply a fibrant replacement functor for $\mathrm{Alg}_{C}$. But every object is fibrant.

For symmetric or orthogonal spectra, the argument goes exactly as in $\S 15$ of [27]. The argument there is only for the commutative algebra operad, but it goes through with no changes for the geometric realization of an arbitrary simplicial operad.

Axiom 5 in all these cases follows from the observation that for cofibrant $X$ (in the positive model category structure where required), the smash product $X^{(n)}$ is actually a free $\Sigma_{n}$-spectrum. For symmetric and orthogonal spectra, see Lemma 15.5 of [27]; for $S$-modules see Theorem III.5.1 of [16].

We would guess this result is also true for symmetric spectra in simplicial sets, but this is not immediately obvious: the case of symmetric spectra in spaces uses that the inclusion of a sphere into a disk is an $N D R$-pair.

The following result is why we put the final axiom into our list 1.4.

1.6 Theorem. Let $C \rightarrow D$ be a morphism of operads in simplicial sets. Then the adjoint pair

$$
f^{*}: \operatorname{Alg}_{C} \rightleftarrows \operatorname{Alg}_{D}: f_{*}
$$

is a Quillen pair. If, in addition, the morphism of operads has the the property that $C(n) \rightarrow D(n)$ is a weak equivalence of spaces for all $n \geq 0$, this Quillen pair is a Quillen equivalence.

Proof. The fact that we have a Quillen pair follows from the fact that the restriction of structure functor (the right adjoint) $f_{*}: \operatorname{Alg}_{D} \rightarrow \operatorname{Alg}_{C}$ certainly preserves weak equivalences and fibrations.

For the second assertion, first note that since $f_{*}$ reflects weak equivalences, we need only show that for all cofibrant $X \in \operatorname{Alg}_{C}$, the unit of the adjunction

$$
X \rightarrow f_{*} f^{*} X=D \otimes_{C} X
$$

is a weak equivalence. If $X=C\left(X_{0}\right)$ is actually a free algebra on a cofibrant spectrum, then this map is exactly the map induced by $f$ :

$$
C\left(X_{0}\right)=\bigvee_{n} C(n)_{+} \wedge_{\Sigma_{n}} X_{0}^{(n)} \rightarrow \bigvee_{n} D(n)_{+} \wedge_{\Sigma_{n}} X_{0}^{(n)}=D\left(X_{0}\right)
$$

For this case, Axiom 5 of 1.4 supplies the result. We now reduce to this case. 
Let $X \in \mathcal{S}^{C}$ be cofibrant. We will make use of an augmented simplicial resolution

$$
P \bullet \longrightarrow X
$$

with the following properties:

i.) the induced map $\left|P_{\bullet}\right| \rightarrow X$ from the geometric realization of $P_{\bullet}$ to $X$ is a weak equivalence;

ii. ) the simplicial $C$-algebra is $s$-free on a set of $C$-algebras $\left\{C\left(Z_{n}\right)\right\}$ where each $Z_{n}$ is a cofibrant spectrum. (The notion of $s$-free was defined at the end of the introduction.)

There are many ways to produce such a $P_{\bullet}$. For example, we could take an appropriate subdivision of a cofibrant model for $X$ in the resolution model category for simplicial $C$-algebras based on the homotopy cogroup objects $C\left(S^{n}\right)$, $-\infty<n<\infty$.

Given $P_{\bullet}$, consider the diagram

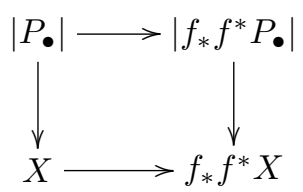

For all $n$, we have an isomorphism

$$
P_{n} \cong C\left(\bigvee_{\phi:[n] \rightarrow[k]} Z_{k}\right)
$$

where $\phi$ runs over the surjections in the ordinal number category. Thus we can conclude that $P_{n} \rightarrow f_{*} f^{*} P_{n}$ is a weak equivalence and that both $P_{\bullet}$ and $f_{*} f^{*} P_{\bullet}$ are Reedy cofibrant. The result now follows from the diagram 1.1.

We now make precise the observation that Theorem 1.6 implies that the notion of, for example, an $E_{\infty}$ ring spectrum is independent of which $E_{\infty}$ operad we choose.

First we recall the Dwyer-Kan [12] classification space in a model category. Let $\mathcal{M}$ be a model category and let $\mathbf{E}$ be a subcategory of $\mathcal{M}$ which has the twin properties that

1.) if $X$ is an object in $\mathbf{E}$ and $Y$ is weakly equivalent to $X$, then $Y \in \mathbf{E}$;

2.) the morphisms in $\mathbf{E}$ are weak equivalences and if $f: X \rightarrow Y$ is a weak equivalence in $\mathcal{M}$ between objects of $\mathbf{E}$, then $f \in \mathbf{E}$.

For example, $\mathbf{E}$ might have the same objects as $\mathcal{M}$ and all weak equivalences, in which case we will write $\mathbf{E}(\mathcal{M})$.

\footnotetext{
${ }^{1}$ Resolution model categories are reviewed in section 3 .
} 
Let $B \mathbf{E}$ denote the nerve of the category $\mathbf{E}$. While the category $\mathbf{E}$ might not be small, one of the theorems of Dwyer and Kan is that it is homotopically small in the sense that each component has the weak homotopy type of a simplicial set; thus, by limiting the objects of interest in some way we obtain a useful weak homotopy type. In fact, there is a formula for this weak homotopy type:

$$
B \mathbf{E} \simeq \coprod_{[X]} B \operatorname{Aut}_{\mathcal{M}}(\mathrm{X})
$$

where $[X]$ runs over the weak homotopy types in $\mathbf{E}$ and $\operatorname{Aut}_{\mathcal{M}}(\mathrm{X})$ is the (derived) monoid of self-weak equivalences of $X$.

To this can be added the following result, immediate from Theorem 1.6.

1.7 Corollary. Let $C \rightarrow D$ be any morphism of simplicial operads so that $C(n) \rightarrow D(n)$ is a weak equivalence of spaces for all $n \geq 0$. Then the natural map induced by restriction of structure

$$
B \mathbf{E}\left(\mathcal{S}^{D}\right) \rightarrow B \mathbf{E}\left(\mathcal{S}^{C}\right)
$$

is a weak equivalence.

1.8 Remark. 1.) Note that Theorem 1.6 and Corollary 1.7 do not require that the operads be cofibrant. Thus, if we define an $E_{\infty}$-operad $C$ to be an operad so that each $C(n)$ is a free and contractible $\Sigma_{n}$-space, then $C$ is weakly equivalent to the commutative monoid operad Comm which is simply a point in each degree. These results then say that the category of $E_{\infty}$-ring spectra (algebras over $C$ ) is Quillen equivalent to the category of commutative $S$-algebras (algebras over Comm).

2.) Let $C \in \mathcal{O}$ be an operad in simplicial sets and let $X$ be a spectrum. We now define the moduli space $C[X]$ of $C$-algebra structures on $X$ by the homotopy pull-back diagram

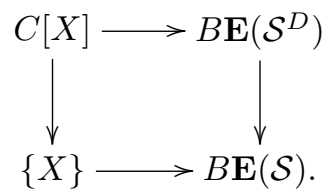

Thus, for example, $C[X]$ is not empty if and only if $X$ has a $C$-algebra structure. Note that Corollary 1.7 implies that $C[X]$ is independent of $C$ up to the naïvest sort of weak equivalence.

In the case of spaces (rather than spectra) Charles Rezk has shown in [33] that $C[X]$ is equivalent to the (derived) space of operad maps from $C$ to the endomorphism operad of $X$. Furthermore, he gives a way of approaching the homotopy type of $C[X]$ using a type of Hochschild cohomology. A similar result is surely true here; see the last sections of [34] for more details on this approach.

Our project is slightly different. Rather than beginning with a spectrum $X$ we will begin with an algebraic model and try to construct $E_{\infty}$-ring spectrum from this data. In effect, we deal directly with $B \mathbf{E}\left(\mathcal{S}^{D}\right)$. 


\section{Simplicial spectra over simplicial operads}

Simplicial objects have often been used to build resolutions and that is our main point here, also. However, given an algebra $X$ in spectra over some operad, we will resolve not only $X$, but the operad as well. The main results of this section are that if $X$ is a simplicial algebra over a simplicial operad $T$ then the geometric realization $|X|$ is an algebra over the geometric realization $|T|$ and, furthermore, that geometric realization preserves level-wise weak equivalences between Reedy cofibrant objects, appropriately defined.

Let's begin by talking about simplicial operads. As mentioned in the previous section, the category of operads $\mathcal{O}$ is a simplicial model category. From this one gets the Reedy model category structure on simplicial operads $s \mathcal{O}([32])$, which are the simplicial objects in $\mathcal{O} .^{2}$ Weak equivalences are level-wise and cofibrations are defined using the latching objects. The Reedy model category structure has the property that geometric realization preserves weak equivalences between cofibrant objects. It also has a structure as a simplicial model category; for example if $T$ is a simplicial operad and $K$ is simplicial set, then

$$
T^{K}=\left\{T_{n}^{K}\right\} .
$$

However, note that this module structure over simplicial sets is inherited from $\mathcal{O}$ and is not the simplicial structure arising externally, as in [31], §II.2.

Let us next spell out the kind of simplicial operads we want. If $E_{*}$ is the homology theory of a homotopy commutative ring spectrum and $C$ is an operad in $\mathcal{O}$, one might like to compute $E_{*} C(X)$. As mentioned in the introduction, this is usually quite difficult, unless $E_{*} X$ is projective as an $E_{*}$ module and $\pi_{0} C(q)$ is a free $\Sigma_{q}$-set for all $q$. Thus we'd like to resolve $C$ using operads of this sort.

If $T$ is a simplicial operad and $E$ is a commutative ring spectrum in the homotopy category of spectra, then $E_{*} T$ is a simplicial operad in the category of $E_{*}$ modules. The category of simplicial operads in $E_{*}$ modules has a simplicial model category structure in the sense of $\S I I .4$ of [31], precisely because there is a free operad functor. Cofibrant objects are retracts of diagrams which are "free" in the sense of [31]; meaning the underlying degeneracy diagram is a free diagram of free operads.

Given an operad $C \in \mathcal{O}$, we'd like to consider simplicial operads $T$ of the following sort:

2.1 Theorem. Let $C \in \mathcal{O}$ be an operad. Then there exists an augmented simplicial operad

$$
T \longrightarrow C
$$

so that

1.) $T$ is Reedy cofibrant as a simplicial operad;

\footnotetext{
${ }^{2}$ These are bisimplicial operads, but when we say simplicial operad, we will mean a simplicial object in $\mathcal{O}$, emphasizing the second (external) simplicial variable as the resolution variable. The first (internal) simplicial variable will be regarded as the geometric variable.
} 
2.) For each $n \geq 0$ and each $q \geq 0, \pi_{0} T_{n}(q)$ is a free $\Sigma_{q}$-set;

3.) The map of operads $|T| \rightarrow C$ induced by the augmentation is a weak equivalence;

4.) If $E_{*} C(q)$ is projective as an $E_{*}$ module for all $q$, then $E_{*} T$ is cofibrant as a simplicial operad in $E_{*}$ modules and $E_{*} T \rightarrow E_{*} C$ is a weak equivalance of operads in that category.

This theorem is not hard to prove, once one has the explicit construction of the free operad; for example, see the appendix to [33]. Indeed, here is a construction: first take a cofibrant model $C^{\prime}$ for $C$. Then, if $F_{\mathcal{O}}$ is the free operad functor on graded spaces, one may take $T$ to be the standard cotriple resolution of $C^{\prime}$. What this theorem does not supply is some sort of uniqueness result for $T$; nonetheless, what we have here is sufficient for our purposes.

Note that if $C$ is the commutative monoid operad, then we can simply take $T$ to be a cofibrant model for $C$ in the category of simplicial operads and run it out in the simplicial (i.e., external) direction. Then $T$ is, of course, an example of an $E_{\infty}$-operad; and $E_{*} T$ will be an $E_{\infty}$-operad in $E_{*}$-modules in the sense of Definition 6.1.

Now fix a simplicial operad $T=\left\{T_{n}\right\}$. Since the free algebra functor $X \mapsto$ $C(X)$ is natural in $X$ and the operad $C$, we see that if $X$ is a simplicial spectrum, so is $T(X)$. Hence a simplicial algebra in spectra over $T$ is a simplicial spectrum $X$ equipped with a multiplication map

$$
T(X) \longrightarrow X
$$

so that the usual associativity and unit diagrams commute. In particular, if $X=$ $\left\{X_{n}\right\}$, then each $X_{n}$ is a $T_{n}$-algebra. Let $s \operatorname{Alg}_{T}$ be the category of simplicial $T$-algebras.

The category $s \operatorname{Alg}_{T}$ is a simplicial model category. Recall that given a morphism of operads $C \rightarrow D$, then the restriction of structure functor $\operatorname{Alg}_{D} \rightarrow$ $\mathrm{Alg}_{C}$ is continuous. This implies that if $K$ is a simplicial set and $X \in s \operatorname{Alg}_{T}$, we may define $X \otimes K$ and $X^{K}$ level-wise; for example,

$$
X \otimes K=\left\{X_{n} \otimes K\right\}
$$

We could use this structure to define a geometric realization functor; however, we prefer to proceed as follows.

If $\mathcal{M}$ is a module category over simplicial sets, then the geometric realization functor $|\cdot|: s \mathcal{M} \rightarrow \mathcal{M}$ has a right adjoint

$$
Y \mapsto Y^{\Delta}=\left\{Y^{\Delta^{n}}\right\} .
$$

where $\Delta^{n}$ is the standard $n$-simplex. In particular, this applies to simplical operads, and we are interested in the unit of the adjunction $T \rightarrow|T|^{\Delta}$. If $C$ is any operad and $Y$ is a $C$-algebra, then for all simplicial sets $K$, the spectrum 
$Y^{K}$ is a $C^{K}$ algebra. From this it follows that $Y^{\Delta}$ is a simplicial $C^{\Delta}$ algebra. Setting $C=|T|$ and restricting structure defines a functor

$$
Y \mapsto Y^{\Delta}: \operatorname{Alg}_{|T|} \longrightarrow s \operatorname{Alg}_{T} .
$$

The result we want is the following.

2.2 Theorem. Let $T$ be a simplicial operad and $X \in s \operatorname{Alg}_{T}$ a simplicial Talgebra. Then the geometric realization $|X|$ of $X$ as a spectrum has a natural structure as a $|T|$ algebra and, with this structure, the functor

$$
X \mapsto|X|
$$

is right adjoint to $Y \mapsto Y^{\Delta}$.

Proof. We know that for an operad $C \in \mathcal{O}$ the forgetful functor from $\operatorname{Alg}_{C}$ to spectra makes geometric realization. Actually, what one proves is that if $X$ is a simplicial spectrum and $C(X)$ is the simplicial $C$-algebra on $X$, then there is a natural (in $C$ and $X$ ) isomorphism

$$
C(|X|) \longrightarrow|C(X)| .
$$

Now use a diagonal argument. If $T$ is a simplicial operad and $X$ is a simplicial spectrum, then

$$
T(X)=\operatorname{diag}\left\{T_{p}\left(X_{q}\right)\right\} .
$$

Since the functor $D \mapsto D(Y)$ is a continuous left adjoint, taking the realization in the $p$-variable yields a simplicial object

$$
\left\{\left|\left\{T_{\bullet}\left(X_{q}\right)\right\}\right|\right\} \cong\left\{|T|\left(X_{q}\right)\right\} .
$$

Now take the realization in the $q$ variable and get

$$
|T(X)| \cong|T|(|X|)
$$

using the fact about the constant case sited above. The result now follows.

In light of Theorem 2.1 and Theorem 1.6, this theorem gives a tool for creating homotopy types of algebras over operads.

The next item to study is the homotopy invariance of the geometric realization functor, in this setting. The usual result has been cited above: realization preserves level-wise weak equivalences between Reedy cofibrant objects. The same result holds in this case, but one must take some care when defining "Reedy cofibrant". The difficulty is this: the definition of Reedy cofibrant involves the latching object, which is the colimit

$$
L_{n} X=\operatorname{colim}_{\phi:[n] \rightarrow[m]} X_{m}
$$

where $\phi$ runs over the non-identity surjections in the ordinal number category. We must define this colimit if each of the $X_{m}$ is an algbera over a different 
operad. The observation needed is the following. Let $S: I \rightarrow \mathcal{O}$ be a diagram of operads. Then an $I$-diagram of $S$-algebras is an $I$-diagram $X: I \rightarrow \mathcal{S}$ of spectra equipped with a natural transformation of $I$-diagrams

$$
S(X) \rightarrow X
$$

satisfying the usual associativity and unit conditions. For example if $I=\Delta^{o p}$ one recovers simplicial $S$-algebras. Call the category of such $\operatorname{Alg}_{S} .{ }^{3}$ Then one can form the colimit operad colim $S=\operatorname{colim}_{I} S$ and there is a constant diagram functor

$$
\operatorname{Alg}_{\text {colim } S} \longrightarrow \operatorname{Alg}_{S}
$$

sending $X$ to the constant $I$-diagram $i \mapsto X$ where $X$ gets an $S_{i}$ structure via restriction of structure along

$$
S_{i} \longrightarrow \operatorname{colim}_{I} S \text {. }
$$

2.3 Lemma. This constant diagram functor has a left adjoint

$$
X \rightarrow \operatorname{colim}_{I} X \text {. }
$$

Despite the notation, $\operatorname{colim}_{I} X$ is not the colimit of $X$ as an $I$ diagram of spectra; indeed, if $X=S(Y)$ where $Y$ is an $I$-diagram of spectra

$$
\operatorname{colim}_{I} X \cong\left(\operatorname{colim}_{I} S\right)\left(\operatorname{colim}_{I} Y\right) .
$$

If $T$ is a simplicial operad we can form the latching object

$$
L_{n} T=\underset{\phi:[n] \rightarrow[m]}{\operatorname{colim}} T_{m} .
$$

There are natural maps $L_{n} T \rightarrow T_{n}$ of operads. If $X$ is a simplicial $T$-algebra we extend this definition slightly and define

$$
L_{n} X=T_{n} \otimes_{L_{n} T} \underset{\phi:[n] \rightarrow[m]}{\operatorname{colim}} X_{m}
$$

where, again, $\phi$ runs over the non-identity surjections in $\Delta$. In short we extend the operad structure to make $L_{n} X$ a $T_{n}$-algebra and the natural map $L_{n} X \rightarrow$ $X_{n}$ a morphism of $T_{n}$-algebras.

With this construction on hand one can make the following definition. Let $T$ be a simplicial operad and $f: X \rightarrow Y$ a morphism of simplicial $T$-algebras. Then $f$ is a level-wise weak equivalence (or Reedy weak equivalence) if each of the maps $X_{n} \rightarrow Y_{n}$ is a weak equivalence of $T_{n}$-algebras - or, by definition, a weak equivalence as spectra. The morphism $f$ is a Reedy cofibration if the morphism of $L_{n} T$-algebras

$$
L_{n} Y \sqcup_{L_{n} X} Y_{n} \longrightarrow Y_{n}
$$

is a cofibration of $T_{n}$-algebras. The coproduct here occurs in the category of $T_{n}$-algebras. The main result is then:

\footnotetext{
${ }^{3}$ This is a slight variation on the notation $s \operatorname{Alg}_{T}$. If $T$ is a simplicial operad, this new notation would simply have us write $\operatorname{Alg}_{T}$ for $s \operatorname{Alg}_{T}$. No confusion should arise.
} 
2.4 Theorem. With these definitions, and the level-wise simplicial structure defined above, the category s $\mathrm{Alg}_{T}$ becomes a simplicial model category. Furthermore

1.) The geometric realization functor $|-|: s \mathrm{Alg}_{T} \rightarrow \mathrm{Alg}_{|T|}$ sends level-wise weak equivalences between Reedy cofibrant objects to weak equivalences; and

2.) if $T$ is Reedy cofibrant as a simplicial operad, then any Reedy cofibration in $s \operatorname{Alg}_{T}$ is a Reedy cofibration of simplicial spectra.

The importance of the second item in this result is that, in light of Theorem 2.2 , one can calculate the homotopy type of the geometric realization of a $T$ algebra entirely in spectra.

\section{Resolutions}

Building on the results of the last section, we'd like to assert the following. Let $X$ be a simplicial algebra over a simplicial operad $T$, and suppose $T$ satisfies all the conclusions of Theorem 2.1. Then there is a simplicial $T$-algbera $Y$ and a morphism of $T$-algebras $Y \rightarrow X$ so that a.) $|Y| \rightarrow|X|$ is a weak equivalence and b.) $E_{*} Y$ is cofibrant as an $E_{*} T$ algebra. The device for this construction is an appropriate Stover resolution ([38],[14],[15]) and, particularly, the concise and elegant paper of Bousfield [9]. ${ }^{4}$ We explain some of the details in this section.

We begin by specifying the building blocks of our resolutions. We fix a spectrum $E$ which is a commutative ring object in the homotopy category of spectra. Let $D(\cdot)$ denote the Spanier-Whitehead duality functor.

3.1 Definition. A homotopy commutative and associative ring spectrum $E$ satisfies Adams's condition if $E$ can be written, up to weak equivalence, as a homotopy colimit of finite cellular spectra $E_{\alpha}$ with the properties that

1.) $E_{*} D E_{\alpha}$ is projective as an $E_{*}$-module; and

1. for every module spectrum $M$ over $E$ the Künneth map

$$
\left[D E_{\alpha}, M\right] \longrightarrow \operatorname{Hom}_{E_{*}-\bmod }\left(E_{*} D E_{\alpha}, M_{*}\right)
$$

is an isomorphism.

This is the condition Adams (following Atiyah) wrote down in [1] to guarantee that the (co-)homology theory over $E$ has Künneth spectral sequences. If $M$ is a module spectrum over $E$, then so is every suspension or desuspension of $M$; therefore, one could replace the source and target of the map in part 2.) of this definition by the corresponding graded objects.

\footnotetext{
${ }^{4}$ Bousfield's paper is written cosimplicially, but the arguments are so categorical and so clean that they easily produce the simplicial objects we require.
} 
Many spectra of interest satisfy this condition; for example, if $E$ is the spectrum for a Landweber exact homology theory, it holds. (This is implicit in [1], and made explicit in [34].) In fact, the result for Landweber exact theories follows easily from the example of $M U$, which, in turn, was Atiyah's original example. See [2]. Some spectra do not satisfy this condition, however - the easiest example is $H \mathbb{Z}$.

We want to use the spectra $D E_{\alpha}$ as detecting objects for a homotopy theory, but first we enlarge the scope a bit.

3.2 Definition. Define $\mathcal{P}(E)=\mathcal{P}$ to be a set of finite cellular spectra so that

1. the spectrum $S^{0} \in \mathcal{P}$ and $E_{*} X$ is projective as an $E_{*}$-module for all $X \in \mathcal{P}$;

2. for each $\alpha$ there is finite cellular spectrum homotopy equivalent to $D E_{\alpha}$ in $\mathcal{P}$;

3. $\mathcal{P}$ is closed under suspension and desuspension;

4. $\mathcal{P}$ is closed under finite wedges; and

5. for all $X \in \mathcal{P}$ and all $E$-module spectra $M$ the Künneth map

$$
[X, M] \longrightarrow \operatorname{Hom}_{E_{*}-\bmod }\left(E_{*} X, M_{*}\right)
$$

is an isomorphism.

The $E_{2}$ or resolution model category which we now describe uses the set $\mathcal{P}$ to build cofibrations in simplicial spectra and, hence, some sort of projective resolutions.

Because the category of spectra has all limits and colimits, the category of simplicial spectra is a simplicial category in the sense of Quillen using external constructions as in §II.4 of [31]. However, the Reedy model category structure on simplicial spectra is not a simplicial model category using the external simplicial structure; for example, if $i: X \rightarrow Y$ is a Reedy cofibration and $j: K \rightarrow L$ is a cofibration of simplicial sets, then

$$
i \otimes j: X \otimes L \sqcup_{X \otimes K} Y \otimes K \rightarrow Y \otimes L
$$

is a Reedy cofibration, it is a level-wise weak equivalence if $i$ is, but it is not necessarily a level-wise weak equivalence if $j$ is.

The following ideas are straight out of Bousfield's paper [9].

3.3 Definition. Let $\operatorname{Ho}(\mathcal{S})$ denote the stable homotopy category.

1.) A morphism $p: X \rightarrow Y$ in $\mathbf{H o}(\mathcal{S})$ is $\mathcal{P}$-epi if $p_{*}:[P, X] \rightarrow[P, Y]$ is onto for each $P \in \mathcal{P}$.

2.) An object $A \in \mathbf{H o}(\mathcal{S})$ is $\mathcal{P}$-projective if

$$
p_{*}:[A, X] \longrightarrow[A, Y]
$$

is onto for all $\mathcal{P}$-epi maps. 
3.) A morphism $A \rightarrow B$ of spectra is called $\mathcal{P}$-projective cofibration if it has the left lifting property for all $\mathcal{P}$-epi fibrations in $\mathcal{S}$.

The classes of $\mathcal{P}$-epi maps and of $\mathcal{P}$-projective objects determine each other; furthermore, every object in $\mathcal{P}$ is $\mathcal{P}$-projective. Note however, that the class of $\mathcal{P}$-projectives is closed under arbitrary wedges. The class of $\mathcal{P}$-projective cofibrations will be characterized below; see Lemma 3.7.

3.4 Lemma. 1.) The category $\operatorname{Ho}(\mathcal{S})$ has enough $\mathcal{P}$-projectives; that is, for every object $X \in \mathbf{H o}(\mathcal{S})$ there is a $\mathcal{P}$-epi $Y \rightarrow X$ with $Y \mathcal{P}$-projective.

2.) Let $X$ be a $\mathcal{P}$-projective object. Then $E_{*} X$ is a projective $E_{*}$-module, and the Künneth map

$$
[X, M] \longrightarrow \operatorname{Hom}_{E_{*}-\bmod }\left(E_{*} X, M_{*}\right)
$$

is an isomorphism for all E-module spectra $M$.

Proof. For part 1.) we can simply take

$$
Y=\bigvee_{P \in \mathcal{P}} \bigvee_{f: P \rightarrow X} P
$$

where $f$ ranges over all maps $P \rightarrow X$ in $\operatorname{Ho}(\mathcal{S})$. Then, for part 2.), we note that the evaluation map

$$
Y=\bigvee_{P \in \mathcal{P}} \bigvee_{f: P \rightarrow X} P \longrightarrow X
$$

has a homotopy section. Then the result follows from the properties of the elements of $\mathcal{P}$.

We now come to the $\mathcal{P}$-resolution model category structure. Recall that a morphism $f: A \rightarrow B$ of simplicial abelian groups is a weak equivalence if $f_{*}: \pi_{*} A \rightarrow \pi_{*} B$ is an isomorphism. Also $f: A \rightarrow B$ is a fibration if the induced map of normalized chain complexes $N f: N A \rightarrow N B$ is surjective in positive degrees. The same definitions apply to simplicial $R$-modules or even graded simplicial $R$-modules over a graded ring $R$. A morphism is a cofibration if it is injective with level-wise projective cokernel.

3.5 Definition. Let $f: X \rightarrow Y$ be a morphism of simplicial spectra. Then

1.) the map $f$ is a $\mathcal{P}$-equivalence if the induced morphism

$$
f_{*}:[P, X] \longrightarrow[P, Y]
$$

is a weak equivalence of simplicial abelian groups for all $P \in \mathcal{P}$;

2.) the map $f$ is a $\mathcal{P}$-fibration if it is a Reedy fibration and $f_{*}:[P, X] \longrightarrow[P, Y]$ is a fibration of simplicial abelian groups for all $P \in \mathcal{P}$; 
3.) the map $f$ is a $\mathcal{P}$-cofibration if the induced maps

$$
X_{n} \sqcup_{L_{n} X} L_{n} Y \longrightarrow Y_{n}, \quad n \geq 0,
$$

are $\mathcal{P}$-projective cofibrations.

Then, of course, the theorem is as follows. Let $s \mathcal{S}_{\mathcal{P}}$ denote the category of simplicial spectra with these notions of $\mathcal{P}$-equivalence, fibration, and cofibration.

3.6 Theorem. With these definitions, the category $s_{\mathcal{P}}$ becomes a simplicial model category.

The proof is given in [9]. We call this the $\mathcal{P}$-resolution model category structure. It is cofibrantly generated; furthermore there are sets of generating cofibrations and generating acyclic cofibrations with cofibrant source An object is $\mathcal{P}$-fibrant if and only if it is Reedy fibrant. The next result gives a characterization of $\mathcal{P}$-cofibrations.

Call a morphism $X \rightarrow Y$ of spectra $\mathcal{P}$-free if it can be written as a composition

$$
X \stackrel{i}{\longrightarrow} X \vee F \stackrel{q}{\longrightarrow} Y
$$

where $i$ is the inclusion of the summand, $F$ is cofibrant and $\mathcal{P}$-projective, and $q$ is an acyclic cofibration. The following is also in [9].

3.7 Lemma. A morphism $X \rightarrow Y$ of spectra is a $\mathcal{P}$-projective cofibration if and only if it is a retract of $\mathcal{P}$-free map.

There are two ways to characterize $\mathcal{P}$-equivalences. The first comes directly from the definition of $\mathcal{P}$-equivalences. If $X \in s \mathcal{S}$ and $P \in \mathcal{P}$, then

$$
[P, X]=\left\{\left[P, X_{n}\right]\right\}
$$

is a simplicial abelian group, and we may define

$$
\pi_{i}(X ; P)=\pi_{i}[P, X]
$$

Then, a morphism is a $\mathcal{P}$-equivalence if and only if it induces an isomorphism on $\pi_{*}(-; P)$ for all $P \in \mathcal{P}$.

There are other homotopy groups. Define "sphere objects" in $s \mathcal{S}$ as follows: let $P \in \mathcal{P}, n \geq 0$, and let $\Delta^{n} / \partial \Delta^{n}$ be the standard simplicial $n$-sphere. As always, $\Delta^{0} / \partial \Delta^{0}=\left(\Delta^{0}\right)_{+}$is the two-point simplicial set. Then the $n$th $P_{-}$ sphere $P \wedge \Delta^{n} / \partial \Delta^{n}$ is defined by the push-out diagram

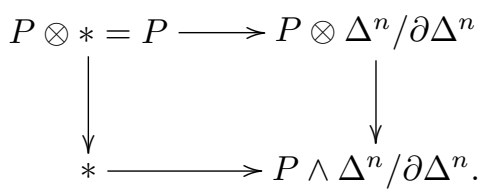


If $X \in s \mathcal{S}$ is a simplicial spectrum, then the mapping $\operatorname{space} \operatorname{map}(P, X)$ is a loop space - in fact, an infinite loop space. Now define the "natural" homotopy groups of a simplicial spectrum $X$ by the formula

$$
\pi_{n}^{\natural}(X ; P)=\left[P \wedge \Delta^{n} / \partial \Delta^{n}, X\right]_{\mathcal{P}} \cong \pi_{n} \operatorname{map}(P, X)
$$

where we take the constant map as the basepoint of the mapping space. The symbol $[,]_{\mathcal{P}}$ refers to morphisms in the homotopy category obtained from the $\mathcal{P}$-resolution model category structure.

The two notions of homotopy groups are related by the spiral exact sequence. Let $\Sigma: \mathbf{H o}(\mathcal{S}) \rightarrow \mathbf{H o}(\mathcal{S})$ be the suspension operator on the homotopy category of spectra.

3.8 Proposition. For all $P \in \mathcal{P}$ and all simplicial spectra $X$, there is a natural isomorphism

$$
\pi_{0}^{\natural}(X ; P) \cong \pi_{0}(X ; P)
$$

and a natural long exact sequence

$$
\begin{aligned}
\cdots \rightarrow \pi_{n-1}^{\natural}(X ; \Sigma P) & \rightarrow \pi_{n}^{\natural}(X ; P) \rightarrow \pi_{n}(X ; P) \\
& \rightarrow \pi_{n-2}^{\natural}(X ; \Sigma P) \rightarrow \cdots \rightarrow \pi_{1}(X ; P) \rightarrow 0 .
\end{aligned}
$$

See [15]. Note that this implies that a morphism of simplicial spectra is a $\mathcal{P}$-equvalence if and only if it induces an isomorphism on $\pi_{*}^{\natural}(-, P)$ for all $P \in \mathcal{P}$.

The long exact sequences of Proposition 3.8 can be spliced together to give a spectral sequence

$$
\pi_{p}\left(X ; \Sigma^{q} P\right) \Longrightarrow \operatorname{colim}_{k} \pi_{k}^{\natural}\left(X ; \Sigma^{p+q-k} P\right) .
$$

using the triangles

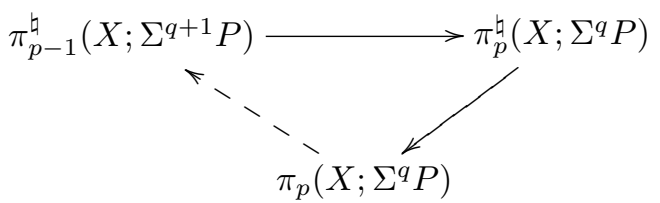

as the basis for an exact couple. Here and below the dotted arrow means a morphism of degree -1 . This is actually a very familiar spectral sequence in disguise.

We may assume that $X$ is Reedy cofibrant, and let $\operatorname{sk}_{n} X$ denote the $n$th skeleton of $X$ as a simplicial spectrum. Then geometric realization makes $\left\{\left|\operatorname{sk}_{n} X\right|\right\}$ into a filtration of $|X|$ and the standard spectral sequence of the geometric realization of simplicial spectrum is gotten by splicing the together the long exact sequences obtained by apply the functor $\left[\Sigma^{p+q} P,-\right]$ to the cofibration sequence

$$
\left|s k_{p-1} X\right| \longrightarrow\left|s k_{p} X\right| \longrightarrow \Sigma^{p}\left(X_{p} / L_{p} X\right)
$$


If we let

$$
\left[\Sigma^{p+q} P,\left|s k_{p} X\right|\right]^{(1)}=\operatorname{Im}\left\{\left[\Sigma^{p+q} P,\left|s k_{p} X\right|\right] \longrightarrow\left[\Sigma^{p+q} P,\left|s k_{p+1} X\right|\right]\right\}
$$

then the first derived long exact sequence of this exact couple is

$$
\left[\Sigma^{p+q} P,\left|s k_{p-1} X\right|\right]^{(1)} \longrightarrow\left[\Sigma^{p+q} P,\left|s k_{p} X\right|\right]^{(1)}
$$

and we obtain the usual spectral seqeunces

$$
\pi_{p}\left(X ; \Sigma^{q} P\right)=\pi_{p}\left[\Sigma^{q} P, X\right] \Longrightarrow\left[\Sigma^{p+q} P,|X|\right] .
$$

Thus the two spectral sequences have isomorphic $E^{2}$-terms. More is true. The next result says that the two exact couples obtained from the triangles of 3.2 and 3.3 are isomorphic; hence, we have isomorphic spectral sequences and we can assert that geometric realization induces an isomorphism

$$
\operatorname{colim}_{k} \pi_{k}^{\natural}\left(X ; \Sigma^{p+q-k} P\right) \stackrel{\cong}{\longrightarrow}\left[\Sigma^{p+q} P,|X|\right] .
$$

3.9 Lemma. Geometric realization induces as isomorphism between the spiral exact sequence

$$
\cdots \rightarrow \pi_{p-1}^{\natural}\left(X ; \Sigma^{q+1} P\right) \rightarrow \pi_{p}^{\natural}\left(X ; \Sigma^{q} P\right) \rightarrow \pi_{p}\left(X ; \Sigma^{q} P\right) \rightarrow \cdots
$$

and the derived exact sequence

$$
\cdots \rightarrow\left[\Sigma^{p+q} P,\left|s k_{p-1} X\right|\right]^{(1)} \longrightarrow\left[\Sigma^{p+q} P,\left|s k_{p} X\right|\right]^{(1)} \longrightarrow \pi_{p}\left[\Sigma^{q} P, X\right] \rightarrow \cdots
$$

Proof. The difficulty is to construct the map of exact sequences inducing an isomorphism $\pi_{p}\left(X ; \Sigma^{q} P\right) \cong \pi_{p}\left[\Sigma^{q} P, X\right]$. Once that is in place, the five lemma and an induction argument show that we must have an isomorphism.

In [15] the spiral exact sequence is obtained by deriving another exact sequence. If $K$ is a finite pointed simplicial set and $X$ is a simplicial spectrum, there is a spectrum $C_{K} X$ characterized by the natural isomorphism

$$
\operatorname{Hom}_{s \mathcal{S}}(Z \wedge K, X) \cong \operatorname{Hom}_{\mathcal{S}}\left(Z, C_{K} X\right)
$$

for all spectra $Z$. In particular, we write

$$
Z_{p} X=C_{\Delta^{p} / \partial \Delta^{p}} X \quad \text { and } \quad C_{p} X=C_{\Delta^{p} / \Delta_{0}^{p}} X
$$

where $\Delta_{0}^{p}$ is the union of all but the 0 th face. If $X$ is Reedy fibrant, there is a fibration sequence

$$
Z_{p} X \longrightarrow C_{p} X \longrightarrow Z_{p-1} X
$$


with maps induced by the cofibration sequence of simplicial sets

$$
\Delta^{p-1} / \partial \Delta^{p-1} \stackrel{d^{0}}{\longrightarrow} \Delta^{p} / \Delta_{0}^{p} \longrightarrow \Delta^{p} / \partial \Delta^{p} .
$$

Then the spiral exact sequence is the first derived sequence of the triangle

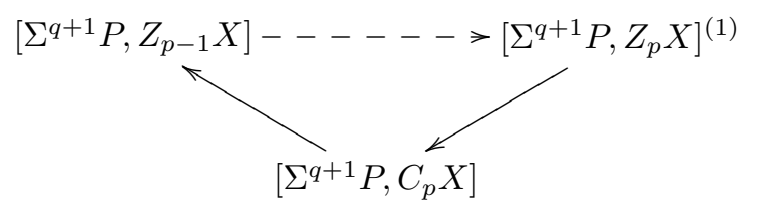

A key calculation is that $\left[\Sigma^{q} P, C_{p} X\right] \cong N_{p}\left[\Sigma^{p}, X\right]$ where $N_{p}(-)$ is the $p$ th group in the normalized chain complex.

Geometric realization induces a function

$$
\operatorname{Hom}_{\mathcal{S}}\left(Z, C_{K} X\right) \cong \operatorname{Hom}_{s \mathcal{S}}(Z \wedge K, X) \longrightarrow \operatorname{Hom}_{\mathcal{S}}(Z \wedge|K|, X) .
$$

This does not induce a map out of the triangle of 3.5; however, after taking first derived triangles, we get a morphism from the triangle of 3.2 to the triangle 3.3 , as required.

3.10 Remark. At this point we can explain one of the reasons for using the models $\mathcal{P}$ to define the resolution model category. Suppose $X \rightarrow Y$ is a weak equivalence between cofibrant objects in the $\mathcal{P}$-resolution model category. Then for each of the spectra $D E_{\alpha}$ we have an isomorphism

$$
f_{*}: \pi_{p}\left(X ; \Sigma^{q} D E_{\alpha}\right) \stackrel{\cong}{\longrightarrow} \pi_{p}\left(Y, \Sigma^{q} D E_{\alpha}\right) .
$$

However, if $E_{*}(-)$ is our chosen homology theory

$$
\begin{aligned}
\pi_{p} E_{q} X & \cong \operatorname{colim}_{\alpha} \pi_{p}\left(E_{\alpha}\right)_{q} X \\
& \cong \operatorname{colim}_{\alpha} \pi_{p}\left[\Sigma^{q} D E_{\alpha}, X\right] \\
& =\operatorname{colim}_{\alpha} \pi_{p}\left(X ; \Sigma^{q} D E_{\alpha}\right) .
\end{aligned}
$$

We note that the spectral sequence of Equation 3.4 is natural in $P$; thus, taking the colimit as this equation, we obtain a spectral sequence

$$
\pi_{p} E_{q} X \Longrightarrow E_{p+q}|X| \text {. }
$$

This is, of course, the standard homology spectral sequence of a simplicial spectrum. In any case, if $X \rightarrow Y$ is an $\mathcal{P}$-equivalence, then we get isomorphic $E_{*}$ homology spectral sequences.

Finally, also note that Lemma 3.9 yields an isomorphism

$$
\operatorname{colim}_{\alpha} \pi_{p}^{\natural}\left(X, \Sigma^{q} D E_{\alpha}\right) \cong \operatorname{Im}\left\{E_{q}\left|\operatorname{sk}_{p} X\right| \rightarrow E_{q}\left|\operatorname{sk}_{p+1} X\right|\right\} .
$$


3.11 Remark. The category $s \mathcal{S}$ of simplicial spectra, and the more structured simplicial spectra defined below have Postnikov sections. That is, for any $X$ in $s \mathcal{S}$ we can produce a morphism of simplicial spectra $X \rightarrow P_{n} X$ so that $\pi_{k}^{\natural}(X ; P) \cong \pi_{k}^{\natural}\left(P_{n} X ; P\right)$ for $P \in \mathcal{P}$ and $k \leq n$, and, in addition, $\pi_{k}^{\natural}\left(P_{n} X ; P\right)=0$ for $k>n$. One way to construct $P_{n} X$ is to define $P_{n} X$ to the colimit of simplicial spectra $P_{n}^{i} X$ where $P_{n}^{0} X$ to be a fibrant model for $X$ and defining $P_{n}^{i} X$ to be a fibrant model for the spectrum simplicial spectrum $Y$ obtained as a push-out

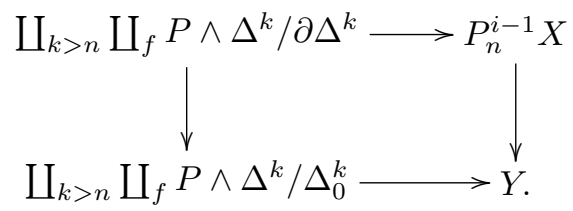

where $f$ runs over all morphisms

$$
f: P \wedge \Delta^{k} / \partial \Delta^{k} \rightarrow P_{n}^{i-1} X
$$

Note that since $s \mathcal{S}_{\mathcal{P}}$ is cofibrantly generated, this can be made natural in $X$. If we are working with algebras in $s \operatorname{Alg}_{T}$ for some simplicial operad $T$, we would simply replace $P \wedge \Delta^{k} / \partial \Delta^{k}$ by $T\left(P \wedge \Delta^{k} / \partial \Delta^{k}\right)$, and so on.

It is worth remarking that one can now recover the universal coefficient theorem of Adams-Atiyah ([1] §III.13) from these constructions. If $X$ is a spectrum, we regard it as constant simplicial spectrum and choose a $\mathcal{P}$-cofibrant replacement $Y$ for $X$. Then the spectral sequence of Equation 3.4, with $P=S^{0}$, implies that $|Y| \simeq X$. The universal coefficient spectral sequence is the Bousfield-Kan spectral sequence of the cosimplicial spectrum $F(Y, M)$ for an $E$-module spectrum $M$ :

$$
\operatorname{Ext}_{E_{*}}^{s}\left(E_{*} X, M_{*+t}\right) \cong \pi^{s} \pi_{-t} F(Y, M) \Longrightarrow \pi_{-t-s} F(|Y|, M) \cong M^{s+t} X .
$$

The $E_{2}$-term is identified using Definition 3.2.4. Here the symbol $M_{*+t}$ means the graded group with $\left(M_{*+t}\right)_{n}=M_{n+t}$.

The $\mathcal{P}$-resolution model category structure can be promoted to a model category for simplicial algebras over a simplicial operad. Fix a simplicial operad $T$ and let $s \operatorname{Alg}_{T}$ be the category of algebras over $T$. This category has an external simplicial structure; indeed, if $K$ is a simplicial set and $X \in s \operatorname{Alg}_{T}$, one has

$$
(X \otimes K)_{n}=\coprod_{K_{n}}{ }^{T_{n}} X_{n} .
$$

The superscript $T_{n}$ is indicates that the coproduct is taken in the category of $T_{n}$ algebras. The simplicial set of maps is defined again by

$$
[n] \mapsto \operatorname{Hom}_{s \operatorname{Alg}_{T}}\left(X \otimes \Delta^{n}, Y\right) .
$$

We say that a morphism $X \rightarrow Y$ of simplicial $T$-algebras is an $\mathcal{P}$-fibration or $\mathcal{P}$-equivalence if the underlying morphism of simplicial spectra is. Then we have the $\mathcal{P}$-resolution model category structure on $s \mathrm{Alg}_{T}$ : 
3.12 Theorem. With these definitions, the category $s \operatorname{Alg}_{T}$ becomes a simplicial model category. Furthermore, for each $X \in s \operatorname{Alg}_{T}$ there is a natural $\mathcal{P}$-equivalence

$$
P_{T}(X) \rightarrow X
$$

so that

1.) $P_{T}(X)$ is cofibrant in the $\mathcal{P}$-resolution model category structure on $s^{T}$;

2.) the underlying degeneracy diagram of $P_{T}(X)$ is of the form $T(Z)$ where $Z$ is free as a degeneracy diagram and each $Z_{n}$ is a wedge of elements of $\mathcal{P}$.

Proof. The existence of the model category structure is the standard lifting argument. In fact, since $s \mathrm{Alg}_{T}$ has a functorial path object and the forgetful functor to $s \mathcal{S}$ creates filtered colimits in $s \mathrm{Alg}_{T}$, we need only supply a $\mathcal{P}$-fibrant replacement functor for $s \operatorname{Alg}_{T}$. However, every Reedy fibrant object in $s \operatorname{Alg}_{T}$ (as in the previous section) will be $\mathcal{P}$-fibrant, and the $s \mathrm{Alg}_{T}$ in its Reedy model category structure is cofibrantly generated, so we can choose a Reedy fibrant replacement functor. This will do the job.

The object $P_{T}(X)$ is produced by taking an appropriate subdivision (for example the big subdivision of [8] §XII.3, Example 3.4) of a cofibrant model for $X$.

Here is how one might use this model category structure. We fix an operad $C \in \mathcal{O}$ and a simplicial resolution $T \rightarrow C$ of $C$ as in Theorem 2.1. If $X$ is an $C$-algebra, then $X$ can be regarded as a constant object in $s \operatorname{Alg}_{T}$ and, hence, we have the resolution $P_{T}(X) \rightarrow X$ of the previous result. Then $P_{T}(X)$ is Reedy cofibrant in $s \mathrm{Alg}_{T}$ and, by Theorem 2.4.2, also Reedy cofibrant as a simplicial spectrum. Thus we can use the spectral sequence of Equation 3.4 with $P=S^{0}$ to show that the natural map

$$
\left|P_{T}(X)\right| \rightarrow X
$$

is a weak equivalence. But also, arguing as in Remark 3.10 we have that the augmentation $P_{T}(X)$ induces an isomorphism

$$
\pi_{*} E_{*} P_{T}(X) \cong E_{*} X .
$$

Finally, if $E_{*} C(n)$ is projective as an $E_{*}$ module for all $n$, then $E_{*} P_{T}(X)$ is a cofibrant $E_{*} T$ algebra. In fact, since each of the spectra $P \in \mathcal{P}$ has the property that $E_{*} X$ is projective as an $E_{*}$-module, Theorems 2.1 and 3.12 imply that there is an isomorphism of underlying degeneracy objects:

$$
E_{*}\left(P_{T}(X)\right) \cong\left(E_{*} T\right)\left(E_{*} Z\right) .
$$

The fact that $E_{*} P_{T}(X)$ is cofibrant can be read off of this equation.

As this discussion indicates, and as the reader may have already suspected, we are not really interested in the $\mathcal{P}$-equivalence classes of objects in simplicial spectra or simplicial $T$-algebras, but certain types of $E_{*}$-equivalences. There is an appropriate model category, and it is a localization of the one supplied in Theorem 3.12. 
3.13 Theorem. The category $s \operatorname{Alg}_{T}$ supports the structure of a cofibrantly generated simplicial model category with

1.) a morphism $f: X \rightarrow Y$ is an $E_{*}$-equivalence if

$$
\pi_{*} E_{*}(f): \pi_{*} E_{*} X \longrightarrow \pi_{*} E_{*} Y
$$

is an isomorphism;

2.) a morphism is an $E_{*}$-cofibration if it is a $\mathcal{P}$-cofibration; and

3.) a morphism is an $E_{*}$-fibration if it has the right lifting property with respect to all morphisms which are at once an $E_{*}$-equivalence and an $E_{*}$ cofibration.

Since every $\mathcal{P}$-equivalence in $s \mathrm{Alg}_{T}$, is an $E_{*}$-equivalence, this model category structure can be produced using the localization technology of Bousfield, et al. The are many minute details, but the technology is now available in [20].

\section{André-Quillen cohomology}

If $A$ is a commutative algebra over a commutative ring $k, M$ an $A$-module and $X \rightarrow A$ a morphism of $k$-algebras, then the André-Quillen cohomology of $X$ with coefficients in $M$ is the non-abelian right derived functors of the functor

$$
X \mapsto \operatorname{Der}_{k}(X, M)
$$

which assigns to $X$ the $A$-module of $k$-derivations from $X$ to $M$. This cohomology has natural generalization to algebras over operads and their modules; indeed, much of the formalism of Quillen's paper [30] goes through without difficulty. This section outlines the details and explains the application to the computation of the homotopy type of the space of maps between structured ring spectra.

It should be said that for someone interested primarily in some $A_{\infty}$ operad that is, in producing associative ring spectra - then the André-Quillen cohomology produced and discussed here is exactly that of the associative algebra case in [30]. It is, except possibly in degree zero, a shift of Hochschild cohomology.

The first part of this section is written algebraically. We fix a commutative ring $k$, possibly graded, and we consider $k$-modules (again possibly graded), operads in $k$-modules, and so on. All tensor products will be over $k$. In our applications $k$ will be $E_{*}$ for some homotopy commutative ring spectrum $E$. Any omitted details can be found in [18].

Let $C$ be an operad in $k$-modules and suppose $A$ is a $C$ algebra. We define what it means for $M$ to be an $A$-module. Let $\Phi(A, M)$ to be the graded $k$ module with

$$
\Phi(A, M)_{n}=\bigoplus_{i} A \otimes \cdots \otimes A \otimes M_{i} \otimes A \otimes \cdots \otimes A
$$


with $M$ appearing once in each summand and then in the $i$ th slot. Note that $\Phi(A, M)_{n}$ has an obvious action of the symmetric group $\Sigma_{n}$. Define

$$
C(A, M)=\bigoplus_{n} C(n) \otimes_{k \Sigma_{n}} \Phi(A, M)_{n}=\bigoplus_{n} C(n) \otimes_{k \Sigma_{n-1}} A^{\otimes(n-1)} \otimes M .
$$

It is an exercise to show that there is a natural ismorphism of bifunctors

$$
C(C(A), C(A, M)) \cong(C \circ C)(A, M))
$$

where $(\cdot) \circ(\cdot)$ is the composition of operads. The $k$-module $M$ is an A-module over $C$ (or simply an $A$-module) if there is a morphism of $k$-modules $\eta$ : $C(A, M) \rightarrow M$ which fits into a coequalizer diagram

$$
C(C(A), C(A, M)) \cong(C \circ C)(A, M)) \underset{d_{1}}{\stackrel{d_{0}}{\rightrightarrows}} C(A, M) \stackrel{\eta}{\longrightarrow} M
$$

where the maps $d_{0}$ and $d_{1}$ are induced by the operad multiplication of $C$, and by $\eta$ and the algebra structure on $A$ respectively. Furthermore, the unit $\mathbf{1} \rightarrow \mathcal{C}$ defines a morphism of $R$-modules $M=\mathbf{1}(A, M) \rightarrow C(A, M)$ which is required to be a section of $\eta$.

We now come to derivations. If $A$ is a commutative $k$-algebra, and $M$ is an $A$-module, we can can form a new commutative algebra over $A$ called $M \rtimes A$, which as an $k$-module is simply $M \oplus A$, but with algebra multiplication

$$
(x, a)(y, b)=(x b+a y, a b) .
$$

The algebra $M \rtimes A$ is a square-zero extension and an abelian object in the category of algebras over $A$; all abelian group objects in this category have this form. It also represents the functor that assigns to an algebra over $A$ the $A$-module of $k$-derivations from $X$ to $M$ :

$$
\operatorname{Der}_{R}(X, M) \cong \operatorname{Alg}_{\text {Comm } / A}(X, M \rtimes A)
$$

where we write Comm for the commutative algebra operad in $K$-modules.

These concepts easily generalize. If $C$ is an operad, $A$ a $C$-algebra and $M$ an $A$-module, define a new $C$-algebra over $A$ called $M \rtimes A$ as follows: as an $k$-module $M \rtimes A$ is simply $M \oplus A$, but the $C$-algebra structure is defined by noting that there is a natural decomposition

$$
C(M \oplus A) \cong E(A, M) \oplus C(A, M) \oplus C(A)
$$

where $E(A, M)$ consists of those summands of $C(M \oplus A)$ with more than one $M$ term. Since $M$ is an $A$-module we get a composition

$$
C(M \oplus A) \rightarrow C(A, M) \oplus C(A) \rightarrow M \oplus A
$$

which defines the $C$-algebra structure on $M \rtimes A$. The algebra $M \rtimes A$ is again a square-zero extension and an abelian object in the category of algebras over $A$; 
again, all abelian objects have this form. This last observation makes it possible to define the category of $A$-modules over $C$ to be the category of abelian $C$ algebras over $A$.

Note that if we are in a graded setting and $M$ is an $A$-module, then the graded object $\Omega^{t} M$ with

$$
\left(\Omega^{t} M\right)_{k}=M_{k+t}
$$

is also an $A$-module. Also, as obvious example of an $A$-module is $A$ itself. We will write

$$
\Lambda_{A}\left(x_{-t}\right)=\Omega^{t} A \rtimes A
$$

by analogy with the exterior algebra that arises in the commutative case.

The object $M \rtimes A$ in the category of $C$-algebras over $A$ represents an abelian group valued functor which we might as well call derivations; in formulas we write

$$
\operatorname{Der}_{C}(X, M) \stackrel{\text { def }}{=} \operatorname{Alg}_{C / A}(X, M \rtimes A)
$$

for all $C$-algebras over $X$. Such a derivation is determined by an $k$-module homomorphism $d: X \rightarrow M$ which fits into an appropriate diagram which reduces to the usual definition of derivation in the commutative or associative algebra case. We invite the reader to fill in the details.

Cohomology in this context should be derived functors of derivations; this immediately leads us to simplicial algebras. We also need simplicial operads.

Thus, we let $C=C$. be simplicial operad in $k$-modules and $s \operatorname{Alg}_{C}$ the category of simplicial algebras over $C$. This is a simplicial category in the external simplicial structure; for example, if $K$ is a simplicial set and $X \in s \operatorname{Alg}_{C}$ then

$$
(A \otimes K)_{n}=\coprod_{K_{n}} A_{n}
$$

with the coproduct in $C_{n}$-algebras. Also, among the morphisms of $s \operatorname{Alg}_{C}$ we single out the free maps: a morphism $X \rightarrow Y$ is free if the underlying morphism of degeneracy diagrams is isomorphic to a map of the form

$$
X \rightarrow X \sqcup C(Z)
$$

where $Z$ is a free degeneracy diagram on a free $R$-module.

The main theorem of [31] §II.4 immediately implies the following:

4.1 Proposition. The $s \mathrm{Alg}_{C}$ has the structure of a simplicial model category with a morphism $f: X \rightarrow Y$

1. a weak equivalence if $\pi_{*} f: \pi_{*} X \rightarrow \pi_{*} Y$ is an isomorphism;

2. a fibration if the induced map $N f: N X \rightarrow N Y$ of normalized chain complexes in $k$-modules is surjective in positive degrees;

3. a cofibration if it is a retract of a free map. 
If $A \in s \operatorname{Alg}_{C}$ then $\pi_{0} A$ is a $\pi_{0} C$-algebra. If $M$ is a $\pi_{0} A$-module (over the operad $\pi_{0} C$ ) then $M$ is an $A_{n}$-module (over $C_{n}$ ) for all $n \geq 0$. Then we can form the simplicial module $K(M, n)$ over $A$ whose normalization $N K(M, n) \cong M$ concentrated in degree $n$. From this object we can form the simplicial $C$-algebra $K_{A}(M, n)=K(M, n) \rtimes A$ over $A$ and, for $X \in s \operatorname{Alg}_{C} / A$ an algebra over $A$ we will define the André-Quillen cohomology of $X$ with coefficients in $M$ by the formula

$$
D_{C}^{n}(X, M) \stackrel{\text { def }}{=}\left[X, K_{A}(M, n)\right]_{s \operatorname{Alg}_{C} / A} \cong \pi_{0} \operatorname{map}_{s \operatorname{Alg}_{C} / A}\left(X, K_{A}(M, n)\right) .
$$

We note immediately that there are natural isomorphisms

$$
D_{C}^{n-i}(X, M) \cong \pi_{i} \operatorname{map}_{s \operatorname{Alg}_{C} / A}\left(X, K_{A}(M, n)\right)
$$

and that, in fact, the collection of $\operatorname{spaces}^{\operatorname{map}_{s} \operatorname{Alg}_{C} / A}\left(X, K_{A}(M, n)\right), n \geq 0$, assemble into a spectrum $\operatorname{hom}_{s} \operatorname{Alg}_{C} / A\left(X, K_{A} M\right)$ so that

$$
D_{C}^{n}(X, M) \cong \pi_{-n} \operatorname{hom}_{s} \operatorname{Alg}_{C} / A\left(X, K_{A} M\right) .
$$

As usual, this cohomology can be written down as the cohomology of a chain complex. To be concrete about this, let us fix some notation. If $C$ is our simplicial operad and $Y$ is a simplicial $C$-algebra over a constant algebra $A$, and if $M$ is an $A$-module, as above, then we have abelian groups

$$
\operatorname{Der}_{C_{n}}\left(Y_{n}, M\right)=\left(\operatorname{Alg}_{C_{n}} / A\right)\left(Y_{n}, M \rtimes A\right) .
$$

Furthermore, if $\phi:[n] \rightarrow[m]$ is a morphism in the ordinal number category, the $Y_{n}$ is a $C_{m}$-algebra by restriction of structure along $\phi^{*}: C_{m} \rightarrow C_{n}$ and then

$$
\phi^{*}: Y_{m} \longrightarrow Y_{n}
$$

is a morphism of $C_{m}$-algebras. Hence we get a map

$$
\operatorname{Der}_{C_{n}}\left(Y_{n}, M\right) \longrightarrow \operatorname{Der}_{C_{m}}\left(Y_{m}, M\right)
$$

and, in fact, $\operatorname{Der}_{C}(Y, M)$ becomes a cosimplicial abelian $k$-module. Then,

$$
D_{C}^{n}(X, M)=H^{n} N \operatorname{Der}_{C}(Y, M)
$$

where $Y$ is some cofibrant model for $X$ and $N$ is the normalization functor from cosimplicial $k$-modules to cochain complexes of $k$-modules. This concept is important enough that we will write

$$
\mathbb{D}_{C}(X, M) \in \mathbf{H o}\left(C^{*} k\right)
$$

for the well-defined object in the derived category of cochain complexes defined by $N \operatorname{Der}_{C}(Y, M)$, with $Y$ a cofibrant model for $X$.

In our applications we will have a homology theory $E_{*}(\cdot)$ and $k=E_{*}$. We will also have a simplicial operad $T$ - that is, a simplicial object in the category 
$\mathcal{O}$ of simplicial operads - so $C=E_{*} T$ and a typical $C$-algebra will be of the form $E_{*} X$ where $X \in s \operatorname{Alg}_{T}$. If $E_{*} E$ if flat over $E_{*}$, this will imply that we are actually working with operads, algebras and so forth in the category of $E_{*} E$-comodules, rather than simply in the more basic category of $E_{*}$-modules. Under appropriate hypotheses - for example, if $E$ satisfies the Adams condition of Definition 3.1 - the $E_{*} E$-comodule version of Proposition 4.2 is true, and one can use this to define André-Quillen cohomology in the category of $E_{*} E$ comodules.

To do this requires a little care, as we are forced to resolve not only algebras, but also the modules; the short reason for this technical difficulty is that not every chain complex of comodules is fibrant. The same problem arose in [23] and our solution is not much different.

To get started, fix a simplicial operad $C$ in $E_{*} E$-comodules and an $\pi_{0} C$ algebra $A$, also all in $E_{*} E$-comodules.

To ease notation, let us abbreviate the extended comodule functor by

$$
\Gamma(M)=E_{*} E \otimes_{E_{*}} M
$$

The functor $\Gamma$ also induces a right adjoint to the forgetful functor from $A$ modules in $E_{*} E$-comodules to $A$-modules. Indeed, if $M$ is an $A$-module, the module structure on $\Gamma(A)$ is determined by the top split row of the diagram

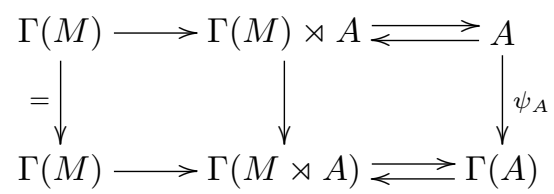

where the right square is a pull-back and where $\psi_{A}$ is the comodule structure map, which, by assumption, is a morphism of algebras. The functor $\Gamma(-)$ thus becomes the functor of a triple on $A$-modules in $E_{*} E$-comodules and for a simplicial $C$-algebra $Y$ in $E_{*} E$ comodules we can form the bicosimplicial $E_{*^{-}}$ module

$$
\operatorname{Der}_{C}\left(Y, \Gamma^{\bullet}(M)\right)=\left\{\operatorname{Der}_{C_{p}}\left(Y_{p}, \Gamma^{q+1}(M)\right)\right\} .
$$

We now write

$$
\mathbb{D}_{C / E_{*} E}(X, M) \in \mathbf{H o}\left(C^{*} E_{*} E\right)
$$

for the object in the derived category of comodules defined by taking $Y$ to be some cofibrant model for $X$ and then taking the total complex of the double normalization of the cosimplicial object $\operatorname{Der}_{C}\left(Y, \Gamma^{\bullet}(M)\right)$. Then we have the André-Quillen cohomology

$$
D_{C / E_{*} E}^{n}(X, M)=H^{n} \mathbb{D}_{E_{*} E / C}(X, M) .
$$


However, with luck, one can reduce the calculation of this more complicated object to the first case. Here is the result we will use. The definitions should make the following results plausible; the proof is in [18].

4.2 Proposition. Let $C$ be a simplicial operad in $E_{*} E$ comodules and $A$ a $\pi_{0} C$ algebra in $E_{*} E$-comodules. If $M$ is a $A$-module in $E_{*}$-modules, then the extended comodule $\Gamma(M)=E_{*} E \otimes_{E_{*}} M$ is an $A$-module in $E_{*} E$-comodules and there is a natural isomorphism for simplicial $C$-algebras $X$ over $A$ in $E_{*} E$-comodules

$$
D_{C / E_{*} E}^{*}\left(X, E_{*} E \otimes_{E_{*}} M\right) \cong D_{C}^{*}(X, M) .
$$

A stronger assertion is true: there is an isomorphism

$$
\mathbb{D}_{C / E_{*} E}\left(X, E_{*} E \otimes_{E_{*}} M\right) \cong \mathbb{D}_{C}(X, M)
$$

in the derived category of $E_{*}$-modules.

With this technology at hand, we can now define a spectral sequence for computing the homotopy groups of the space of maps between two structured ring spectra. We fix a commutative $S$-algebra $E$; that is an algebra in spectra over the commutative algebra operad in $\mathcal{O}$. Suppose further that $E$ satisfies the Adams condition of Definition 3.1. Let $\mathcal{F}$ be one of either the associative algebra operad or the commutative algebra operad in $\mathcal{O} .^{5}$ Now suppose $X$ is an $\mathcal{F}$-algebra in spectra; thus, $X$ is either an associative $S$-algebra or a commutative $S$-algebra. Then $E_{*} X$ is an algebra over the operad $E_{*} \mathcal{F}$ in $E_{*} E$-comodules.

Now let $\phi: X \rightarrow Y$ be a morphism of $\mathcal{F}$-algebras is spectra. This amounts to choosing a basepoint $\phi \in \operatorname{map}_{\operatorname{Alg}_{\mathcal{F}}}(X, Y)$ for the space of $\mathcal{F}$-algebra maps from $X$ to $Y$. The induced map

$$
E_{*} \phi: E_{*} X \longrightarrow E_{*} Y
$$

makes $E_{*} Y$ into an $E_{*} X$ module over the operad $E_{*} \mathcal{F}$, all in $E_{*} E$-comodules. Let $T \rightarrow \mathcal{F}$ be the resolution of operads supplied by Theorem 2.1. Also, for any spectrum $Y$, let $Y_{E}$ denote the $E$-completion of $Y$, defined as the total space of the cobar complex:

$$
Y_{E} \stackrel{\text { def }}{=} \operatorname{Tot}\left(E^{\bullet} \wedge Y\right) \text {. }
$$

Since $E$ is a commutative $S$-algebra, $Y_{E}$ is an $\mathcal{F}$-algebra if $Y$ is an $\mathcal{F}$-algebra.

4.3 Theorem. Let $\phi: X \rightarrow Y$ be a morphism of $\mathcal{F}$-algebras and let $E$ be a commutative $S$-algebra. Then there is a second quadrant spectral sequence abutting to

$$
\pi_{t-s}\left(\operatorname{map}_{\operatorname{Alg}_{\mathcal{F}}}\left(X, Y_{E}\right), \phi\right)
$$

with $E^{2}$ term

$$
E_{2}^{0,0}=\operatorname{Hom}_{E_{*}} \mathcal{F} / E_{*} E\left(E_{*} X, E_{*} Y\right)
$$

and

$$
E_{2}^{s, t}=D_{E_{*} T / E_{*} E}^{s}\left(E_{*} X, \Omega^{t} E_{*} Y\right) \quad t>0 .
$$

\footnotetext{
${ }^{5}$ The reader sensitive to generalization will note that this restriction is only aesthetics. A general operad in $\mathcal{O}$ will do.
} 
Of course, the $E_{2}^{0,0}$ term is either the associative or commutative algebra maps (in $E_{*} E$-comodules) from $E_{*} X$ to $E_{*} Y$.

This is a Bousfield-Kan spectral sequence, as we will see in the next paragraph. The standard references are [8] and [7]. The latter work, for example, implies the following result:

4.4 Corollary. There are succesively defined obstructions to realizing a map $f \in \operatorname{Hom}_{E_{*}} \mathcal{F} / E_{*} E\left(E_{*} X, E_{*} Y\right)$ in the groups

$$
D_{E_{*} T / E_{*} E}^{s+1}\left(E_{*} X, \Omega^{s} E_{*} Y\right) \quad s \geq 1 .
$$

In particular, if these groups are all zero, then the Hurewicz map

$$
\pi_{0}\left(\operatorname{map}_{\operatorname{Alg}_{\mathcal{F}}}\left(X, Y_{E}\right)\right) \rightarrow \operatorname{Hom}_{E_{*} \mathcal{F}}\left(E_{*} X, E_{*} Y\right)
$$

is surjective. If, in addition, the groups

$$
D_{E_{*} T / E_{*} E}^{s}\left(E_{*} X, \Omega^{s} E_{*} Y\right)=0
$$

for $s \geq 1$, the Hurewicz map of Equation 4.6 is a bijection.

The spectral sequence of Theorem 4.3 is constructed as follows. We may regard $X$ as a constant simplicial $T$-algebra and take the simplicial resolution in $T$-algebras $P_{T}(X) \rightarrow X$ guaranteed by Theorem 3.12. In addition, since $E$ is a commutative $S$-algebra, the cobar complex

$$
Y \rightarrow E^{\bullet} \wedge Y=\left\{E^{q+1} \wedge Y\right\}
$$

is a cosimplicial $\mathcal{F}$-algebra. We then obtain a cosimplicial space

$$
\begin{aligned}
\operatorname{map}_{s \operatorname{Alg}_{T}}\left(P_{T}(X), E^{\bullet} \wedge Y\right) & =\left\{\operatorname{map}_{\operatorname{Alg}_{T_{s}}}\left(P_{T}(X)_{s}, E^{s+1} \wedge Y\right)\right\}^{s} \\
& \cong\left\{\operatorname{map}_{\operatorname{Alg}_{\mathcal{F}}}\left(\mathcal{F} \otimes_{T_{s}} P_{T}(X)_{s}, E^{s+1} \wedge Y\right)\right\}^{s}
\end{aligned}
$$

and the map $\phi: X \rightarrow Y$ supplies this with the basepoint. The Bousfield-Kan spectral sequence now reads

$$
\pi^{s} \pi_{t} \operatorname{map}_{\mathrm{Alg}_{T}}\left(P_{T}(X), E^{\bullet} \wedge Y\right) \Longrightarrow \pi_{t-s} \text { TOT } \operatorname{map}_{\mathrm{Alg}_{T}}\left(P_{T}(X), Y\right)^{\bullet}
$$

One uses standard adjunction arguments, Theorem 1.6, Theorem 2.1, and Theorem 2.2 to show

$\operatorname{Toт} \operatorname{Alg}_{T}\left(P_{T}(X), E^{\bullet} \wedge Y\right) \cong \operatorname{Alg}_{|T|}\left(\left|P_{T}(X)\right|, \operatorname{ToT}\left(E^{\bullet} \wedge Y\right)\right) \simeq \operatorname{Alg}_{\mathcal{F}} \mathcal{F}\left(X, Y_{E}\right)$

We then must identify the $E_{2}$-term. Let's abbreviate $E^{(q+1)} \wedge Y$ as $E^{(q)} Y$. Since the cosimplicial space in question is the diagonal of the bicosimplicial space

$$
\left\{\operatorname{map}_{\operatorname{Alg}_{T_{p}}}\left(P_{T}(X)_{p}, E^{(q)} Y\right)\right\}
$$


the $E_{2}$ term can be computed as the cohomology of the total complex of the double normalization of

$$
\left\{\pi_{t} \operatorname{map}_{\mathrm{Alg}_{T_{p}}}\left(P_{T}(X)_{p}, E^{(q)} Y\right)\right\} .
$$

If we let $Y^{S^{t}}$ denote the spectrum of maps from the space $S^{t}$ to the spectrum $Y$, then $Y^{S^{t}}$ has a natural structure of an $\mathcal{F}$-algebra. Thus, because of Theorem 3.12 , the choice of the spectra used to build $\mathcal{P}$-resolutions (Definition 3.2) and the conditions on the operads $T_{s}$ from Theorem 2.1, we have, for $t>0$ :

$$
\begin{aligned}
& \pi_{t} \operatorname{map}_{\operatorname{Alg}_{T_{p}}\left(P_{T}(X)_{p}, E^{(q)}(Y)\right)}=\pi_{t} \operatorname{map}_{\operatorname{Alg}_{T_{p}}}\left(T_{p}\left(Z_{p}\right), E^{(q)}(Y)\right) \\
&=\pi_{t} \operatorname{map}_{\mathcal{S}}\left(Z_{p}, E^{(q)}(Y)\right) \\
&=\operatorname{Hom}_{E_{*} E}\left(E_{*} Z_{p}, E_{*}\left(E^{(q)}(Y)^{S^{t}}\right)\right) \\
&=\operatorname{Hom}_{E_{*} T_{p} / E_{*} E}\left(E_{*} T_{p}\left(E_{*} Z_{p}\right), \Lambda_{E_{*} E^{(q)}(Y)}\left(x_{t}\right)\right) \\
&=\operatorname{Der}_{E_{*} T_{p} / E_{*} E}\left(E_{*} T_{p}\left(E_{*} Z_{p}\right), \Omega^{t} E_{*} E^{(q)} Y\right) .
\end{aligned}
$$

In short, we obtain exactly the complex $\mathbb{D}_{E_{*} T / E_{*} E}\left(E_{*} X, \Omega^{t} E_{*} Y\right)$ used to define André-Quillen cohomology. See Equation 4.4.

As an amusing reduction of this theory, one can consider the case of the unit operad 1 in place of the commutative or associative algebra operad. An algebra over $\mathbf{1}$ is simply a spectrum, and an $E_{*} \mathbf{1}$-algebra is an $E_{*} E$-comodule. The formalism carries over and the spectral sequence of Theorem 4.3 becomes the the Adams-Novikov spectral sequence

$$
\operatorname{Ext}_{E_{*} E}^{s}\left(E_{*} X, \Omega^{t} E_{*} Y\right) \Longrightarrow \pi_{t-s} \operatorname{map}_{\mathcal{S}}\left(X, Y_{E}\right) \cong\left[\Sigma^{t-s} X, Y_{E}\right]
$$

and the obstructions of Corollary 4.4 to realizing an $E_{*} E$-comodule map $E_{*} X \rightarrow$ $E_{*} Y$ lie in

$$
\operatorname{Ext}_{E_{*} E}^{s+1}\left(E_{*} X, \Omega^{s} E_{*} Y\right) \quad s \geq 1 .
$$

If $Y$ is an $E$-module, then the cobar complex $E^{\bullet} \wedge Y$ has a contraction; in particular, $Y=Y_{E}$. We would expect a corresponding simplification of the spectral sequence of Theorem 4.3. Indeed, the André-Quillen cohomology groups simplify: we need only use the derived functors of derivations in $E_{*^{-}}$ modules. We can also weaken the assumption that $E$ be a commutative $S$ algebra. The result then reads:

4.5 Theorem. Suppose that $E$ is a homotopy commutative ring spectrum, satisfying the Adams condition of Definition 3.1 and let $\phi: X \rightarrow Y$ be a morphism of $\mathcal{F}$-algebras in spectra. Then there is a second quadrant spectral sequence abutting to

$$
\pi_{t-s}\left(\operatorname{map}_{\operatorname{Alg}_{\mathcal{F}}}(X, Y), \phi\right)
$$


with $E_{2}$ term

$$
E_{2}^{0,0}=\operatorname{Hom}_{E_{*} \mathcal{F}}\left(E_{*} X, Y_{*}\right)
$$

and

$$
E_{2}^{s, t}=D_{E_{*} T}^{s}\left(E_{*} X, \Omega^{t} Y_{*}\right) \quad t>0 .
$$

Here the $E_{2}^{0,0}$-term is the set of $E_{*} \mathcal{F}$-algebra morphisms from $E_{*} X$ to $Y_{*}$.

The proof is identical to the proof of Theorem 4.3; the relevant cosimplicial space is

$$
\operatorname{map}_{\mathrm{Alg}_{T}}\left(P_{T}(X), Y\right)=\left\{\operatorname{map}_{\mathrm{Alg}_{T_{s}}}\left(P_{T}(X)_{s}, Y\right)\right\}^{s} .
$$

Because we have a cosimplicial space, we again have obstructions to realizing maps. In fact, there are succesively defined obstructions in

$$
D_{E_{*} T}^{s+1}\left(E_{*} X, \Omega^{s} Y_{*}\right) \cong D_{E_{*} T / E_{*} E}^{s+1}\left(E_{*} X, \Omega^{s} E_{*} Y\right), \quad n \geq 1
$$

to the realization of a map in $\operatorname{Hom}_{E_{*} \mathcal{F} / E_{*} E}\left(E_{*} X, E_{*} Y\right)$.

\section{The moduli space of realizations}

We now fix a homotopy commutative ring spectrum $E$ satisfying the Adams condition of Definition 3.1. Let $\mathcal{F}$ be an operad in $\mathcal{O}$ and suppose that $A$ is an $E_{*} \mathcal{F}$-algebra in $E_{*} E$-comodules. The purpose of this section is to discuss the homotopy type of the space $\mathcal{T} \mathcal{M}(A)$ of realizations of $A$ in $\operatorname{Alg}_{\mathcal{F}}$. In practice, of course, $\mathcal{F}$ is either the associative or commutative monoid operad and, hence, $A$ is an associative or commutative algebra in $E_{*} E$-comodules. The method here is exactly that of $[6]$.

By definition, $\mathcal{T} \mathcal{M}(A)$ is the nerve (or classifying space) of the category $\mathcal{E}(A)$ with objects the $\mathcal{F}$-algebra spectra $X$ with $E_{*} X \cong A$ as $E_{*} \mathcal{F}$-algebras and morphisms are $E_{*}$-isomorphisms. ${ }^{6}$ As in section 1, the Dwyer-Kan decomposition of $\mathcal{T} \mathcal{M}(A)$ supplies a weak equivalence

$$
\mathcal{T} \mathcal{M}(A) \simeq \coprod_{[X]} B \operatorname{Aut}(X)
$$

where $X$ ranges over the $E_{*}$-equivalence classes of realizations of $A$ and $\operatorname{Aut}(X)$ is the derived space of self-equivalences of $X$ in the $E_{*}$-local model category structure on $\operatorname{Alg}_{\mathcal{F}}$. It is worth emphasizing that this result uses the identification

$$
B \operatorname{Aut}(X) \simeq \mathcal{M}(X)
$$

where $\mathcal{M}(X)$ is the nerve of the category with objects $Y \in \operatorname{Alg}_{\mathcal{F}}$ so that there a chain of $E_{*}$-isomorphisms in $\operatorname{Alg}_{\mathcal{F}}$ between $Y$ and $X$. The morphisms are $E_{*}$-isomorphisms in $\operatorname{Alg}_{\mathcal{F}}$.

The initial question, of course, is whether $\mathcal{T} \mathcal{M}(A)$ is non-empty.

We now decompose $\mathcal{T} \mathcal{M}(A)$. As always, we will let $T \rightarrow \mathcal{F}$ be a simplicial resolution of the sort supplied by Theorem 2.1.

\footnotetext{
${ }^{6}$ The isomorphism $E_{*} X \cong A$ is not part of the data.
} 
5.1 Definition. Let $X \in s \operatorname{Alg}_{T}$ be a simplicial $T$-algebra. We say that $X$ is a potential $n$-stage for the $E_{*} \mathcal{F}$-algebra $A$ if

1. $\pi_{0} E_{*} X$ is isomorphic to $A$ as an $E_{*} \mathcal{F}$-algebra in $E_{*} E$-comodules;

2. $\pi_{i} E_{*} X=0$ for $1 \leq i \leq n+1$; and

3. For all $P \in \mathcal{P}$, the groups $\pi_{i}^{\natural}(X ; P)=0$ for $i>n$.

The partial moduli space $\mathcal{T} \mathcal{M}_{n}(A)$ is defined to be the moduli space of all simplicial $T$-algebras which are potential $n$-stages for $A$. The weak equivalences are the simplicial $E_{*}$-equivalences of Section 4 .

It follows from the spiral exact sequence of Proposition 3.8 that a potential $n$-stage $X$ for $A$ has

$$
\pi_{i} E_{*} X \cong \begin{cases}A & i=0 \\ \Omega^{n+1} A & i=n+2 \\ 0 & i \neq 0, n+2 .\end{cases}
$$

Furthermore, by the spiral exact sequence or, more exactly, the isomorphism 3.7, the $A$-module structure on $\pi_{n+1} E_{*} X$ is the evident shifted $A$-module structure. The same calculation shows that for the natural homotopy groups

$$
\pi_{i}^{\natural} E_{*} X \stackrel{\text { def }}{=} \operatorname{colim} \pi_{i}^{\natural}\left(X, \Sigma^{*} D E_{\alpha}\right) \cong \begin{cases}\Omega^{i} A & 0 \leq i \leq n ; \\ 0 & i>n .\end{cases}
$$

Again, the $A$-module structure on $\pi_{i}^{\natural} E_{*} X$ is the evident shifted module structure.

Definition 5.1 makes sense for $n=\infty$ : a potential $\infty$-stage is simply an object $X \in s \operatorname{Alg}_{T}$ so that $\pi_{0} E_{*} X \cong A$ and $\pi_{i} E_{*} X=0$ for $i>0$. Let $\mathcal{T} \mathcal{M}_{\infty}(A)$ be the resulting moduli space.

Theorem 2.2 and the spectral sequence of 3.4 imply that geometric realization defines a map

$$
|-|: \mathcal{T} \mathcal{M}_{\infty}(A) \longrightarrow \mathcal{T} \mathcal{M}(A)
$$

and the Postnikov stage construction of Remark 3.11 implies that there are maps

$$
\mathcal{T M}_{n}(A) \longrightarrow \mathcal{T M}_{m}(A) ; \quad 0 \leq m<n \leq \infty .
$$

Here is the first part of the decomposition result.

5.2 Proposition. The map induced by geometric realization

$$
|-|: \mathcal{T} \mathcal{M}_{\infty}(A) \longrightarrow \mathcal{T} \mathcal{M}(A)
$$

is a weak equivalence. Furthermore the map

$$
\mathcal{T} \mathcal{M}_{\infty}(A) \longrightarrow \operatorname{holim}_{n<\infty} \mathcal{T} \mathcal{M}_{n}(A)
$$

is a weak equivalence. 
Proof. The first assertion is formal. Compare Theorem 9.3 of [6]. The second assertion is not formal; however, it follows from the main theorem of [12]. Compare Theorem 9.4 of [6].

The next step is to investigate the tower $\left\{\mathcal{T} \mathcal{M}_{n}(A)\right\}$. To do this we will identify the bottom space as a $K(G, 1)$, then tell how to pass from the $(n-$ $1)$ st stage to the $n$th stage using André-Quillen cohomology. We begin by constructing the 0 -stage; in particular, we show $\mathcal{T} \mathcal{M}_{0}(A) \neq \phi$.

5.3 Definition. A simplicial $T$-algebras is said to be of type $B_{A}$ if

1.) $\pi_{0} E_{*} X \cong A$ as an $E_{*} \mathcal{F}$-algebra in $E_{*} E$ comodules; and

2.) for $Y \in s \operatorname{Alg}_{T}$, the natural map

$$
[Y, X]_{s \operatorname{Alg}_{T}} \longrightarrow \operatorname{Hom}_{E_{*}} \mathcal{F} / E_{*} E\left(\pi_{0} E_{*} Y, A\right)
$$

is an isomorphism. Here the homotopy classes of maps are in the $E_{*}$-local homotopy category of $s \mathrm{Alg}_{T}$.

We write $B_{A}$ for any of the (essentially unique) objects of type $B_{A}$ and, if we need to, will assume $B_{A}$ is $E_{*}$-local without saying so.

Simplicial $T$-algebras of type $B_{A}$ exist. This can be seen by a generators and relations argument or by some generalized Brown representability theorem. See [19].

5.4 Remark. We have that for any simplicial $T$-algebra $X$ of type $B_{A}$ that

$$
\pi_{i}^{\natural}(X ; P)= \begin{cases}\operatorname{Hom}_{E_{*} E}\left(E_{*} P, A\right) & i=0 ; \\ 0 & i>0 .\end{cases}
$$

Thus a simplicial spectrum of type $B_{A}$ is potential 0-stage for $A$. Furthermore, the spiral exact sequence implies

$$
\pi_{i} E_{*} X \cong \begin{cases}A & i=0 \\ \Omega A & i=2 \\ 0 & i \neq 0,2 .\end{cases}
$$

The following result says, among other things, that there is a unique potential 0 -stage of $A$ up to $E_{*}$-equivalence and that it is of type $B_{A}$.

5.5 Proposition. Let $\operatorname{Aut}(A)$ denote the discrete group of automorphisms of the $E_{*} \mathcal{F}$-algebra $A$ in $E_{*} E$-comodules. Then there is a natural weak equivalence

$$
\mathcal{T} \mathcal{M}_{0}(A) \cong B \operatorname{Aut}(A) .
$$

Proof. Fix a choice $B_{A}$ of an $E_{*}$-local space of type $B_{A}$. Let $X$ be a potential 0 -stage for $A$. Then a choice of isomorphism $\pi_{0} E_{*} X \cong A$ defines a morphism in $s \operatorname{Alg}_{T}$

$$
X \longrightarrow B_{A}
$$


which defines an isomorphism on $\pi_{*} E(-)$ by the spiral exact sequence. Thus $\mathcal{T} \mathcal{M}_{0}(A)$ is connected and, by the Dwyer-Kan analysis (5.1)

$$
\mathcal{T M}_{0}(A)=B \operatorname{Aut}\left(B_{A}\right) .
$$

But it is an easy calculation that

$$
\pi_{n} \operatorname{Aut}\left(B_{A}\right) \cong \begin{cases}\operatorname{Aut}(A) & n=0 ; \\ 0 & n \neq 0 .\end{cases}
$$

To pass between the various stages of the tower, we need to know that André-Quillen cohomology is representable in the homotopy category of $s \operatorname{Alg}_{T}$. Specifically, we have the following ideas.

5.6 Definition. Let $A$ be an $E_{*} \mathcal{F}$-algebra in $E_{*} E$-comodules and let $M$ be an $A$-module, also in $E_{*} E$-comodules. We say that a map $X \rightarrow Y$ in $s \operatorname{Alg}_{T}$ is of type $B_{A}(M, n), n \geq 1$ if

1.) $X$ is of type $B_{A}$ and the induced map

$$
\pi_{i} E_{*} X \rightarrow \pi_{i} E_{*} Y
$$

is an isomorphism for $i<n$;

2.) $\pi_{n} E_{*} Y \cong M$ as a $\pi_{0} E_{*} Y \cong A$ module; and

3.) $\pi_{i}^{\natural}(Y ; P)=0$ if $i>n$.

We may abuse notation and refer to the simplicial $T$-algebra $Y$ as being of type $B_{A}(M, n)$. Again, it is possible to construct such objects by a generators and relations argument, or by Brown representability using the evident homotopy characterization supplied by Proposition 5.7 below.

We would like to give a homotopical interpretation of the simplicial $T$ algebras of type $B_{A}(M, n)$; in fact, such objects will - in some sense - represent the functor

$$
Z \mapsto D_{E_{*} T / E_{*} E}^{n}\left(E_{*} Z, M\right) .
$$

The exact result is below in Proposition 5.7, but to get there requires some preliminaries.

If $X \rightarrow Y$ is of type $B_{A}(M, n)$, we may assume that $X$ is $E_{*}$-fibrant and that the map from $X$ to $Y$ is a cofibration to an $E_{*}$-fibrant object - and we may make this assumption without repeating it and then we will write

$$
B_{A} \longrightarrow B_{A}(M, n)
$$

for such a map. If we suppose $n \geq 2$, then the spiral exact sequence implies that

$$
\pi_{i} E_{*} B_{A}(M, n) \cong \pi_{i} E_{*} B_{A} \times \begin{cases}M & i=n \\ \Omega M & i=n+2 \\ 0 & i \neq n, n+2\end{cases}
$$


In particular, we get a natural isomorphism $\pi_{0} E_{*} B_{A}(M, n) \cong A$ and then Remark 5.3 supplies a map $B_{A}(M, n) \rightarrow B_{A}$ so that the composite $X \rightarrow Y \rightarrow B_{A}$ is an $E_{*}$-equivalence. In this way we will regard $B_{A}(M, n)$ as an object over $B_{A}$.

Because of the isomorphism of Equation 5.3, the simplicial $E_{*} T$-algebra $E_{*} B_{A}(M, n)$ is not weakly equivalent to $K_{A}(M, n)=K(M, n) \rtimes A$ in the category of simplicial $E_{*} T$-algebras over $E_{*} E$-comodules. However, there is a natural map of $E_{*} T$ algebras

$$
\epsilon: E_{*} B_{A}(M, n) \longrightarrow K_{A}(M, n)
$$

over the constant simplicial $E_{*} T$-algebra $A$. This we now produce.

Let $C$ be the push-out in $s \operatorname{Alg}_{E_{*} E / E_{*} T}$ of the two-source

$$
E_{*} B_{A}(M, n) \longleftarrow E_{*} B_{A} \longrightarrow \pi_{0} E_{*} E_{*} B_{A} \cong A .
$$

Then Equation 5.3 implies that the $(n+1)$ st Postnikov section $P_{n+1} C$ of $C$ in $s \operatorname{Alg}_{E_{*} E / E_{*} T}$ has the property that

$$
\pi_{i} P_{n+1} C \cong \begin{cases}A & i=0 \\ M & i=n \\ 0 & i \neq 0, n .\end{cases}
$$

This alone is not enough to identify the homotopy type of $P_{n+1} C$. However the map

$$
A \cong \pi_{0} E_{*} B_{A} \rightarrow P_{n+1} C
$$

is a section of the map $P_{n+1} C \rightarrow \pi_{0} P_{n+1} C \cong A$; hence $P_{n+1} C$ is canonically weakly equivalent to $K_{A}(M, n)$, and the composition

$$
E_{*} B_{A}(M, n) \rightarrow C \rightarrow P_{n+1} C
$$

is a model for the morphism $\epsilon$ of Equation 5.4.

5.7 Proposition. Let $B_{A} \rightarrow B_{A}(M, n)$ be of type $B_{A}(M, n)$ and suppose $n \geq$ 2. Let $X \in s \mathrm{Alg}_{T}$ and suppose a morphism of $\mathcal{F}$-algebras in $E_{*} E$-comodules $\pi_{0} E_{*} X \rightarrow A$ is represented by a map $f: X \rightarrow B_{A}$. Then the morphism of simplicial $E_{*} T$-algebras

$$
\epsilon: E_{*} B_{A}(M, N) \rightarrow K_{A}(M, n)
$$

induces a natural weak equivalence

$$
\operatorname{map}_{s \operatorname{Alg}_{T} / B_{A}}\left(X, B_{A}(M, n)\right) \stackrel{\simeq}{\longrightarrow} \operatorname{map}_{s \operatorname{Alg}_{E_{*} T / E_{*} E} / A}\left(E_{*} X, K_{A}(M, n)\right) .
$$

In particular

$$
\pi_{i} \operatorname{map}_{s \operatorname{Alg}_{T} / B_{A}}\left(X, B_{A}(M, n)\right) \cong D_{E_{*} T / E_{*} E}^{n-i}\left(E_{*} X, M\right) .
$$


Proof. We have a natural map induced by $\epsilon$. Since both source and target take homotopy colimits to homotopy limits, it is sufficient to check the result for objects of the form $X=T(P \otimes K)$ where $P \in \mathcal{P}$ and $K$ is a simplicial set. Inducting over the skeleta of $K$, we find it is sufficient to check the result for objects of the form $T\left(P \otimes \partial \Delta^{n}\right)$ equipped with some choice of map

$$
E_{*} T\left(P \otimes \partial \Delta^{n}\right) \rightarrow \pi_{0} E_{*} T\left(P \otimes \partial \Delta^{n}\right) \cong E_{*} \mathcal{F}\left(E_{*} P\right) \rightarrow A .
$$

But the objects of type $B_{A}(M, n)$ are built exactly so the result holds in this case. For more details see Proposition 8.7 of [6].

To shorten notation, let us write

$$
\mathcal{H}^{n}(A, M) \stackrel{\text { def }}{=} \operatorname{map}_{s \operatorname{Alg}_{E_{*} T / E_{*} E} / A}\left(E_{*} X, K_{A}(M, n)\right) .
$$

Let $\operatorname{Aut}(A, M)$ of be the group of automorphisms of the pair $(A, M)$. Then $\operatorname{Aut}(A, M)$ acts in a natural way on the space $\mathcal{H}^{n}(A, M)$; let $\hat{\mathcal{H}}^{n}(A, M)$ denote the Borel construction. The space $\mathcal{H}^{n}(A, M)$ has a basepoint given by

$$
0 \in \pi_{0} \mathcal{H}^{n}(A, M)=D_{E_{*} T / E_{*} E}^{n}(A, M) .
$$

There is a choice of representative for 0 which is invariant under the action of $\operatorname{Aut}(A, M)$; therefore we get an induced map

$$
B \operatorname{Aut}(A, M) \rightarrow \hat{\mathcal{H}}^{n}(A, M)
$$

5.8 Theorem. For all $n \geq 1$ there is a homotopy pull-back diagram

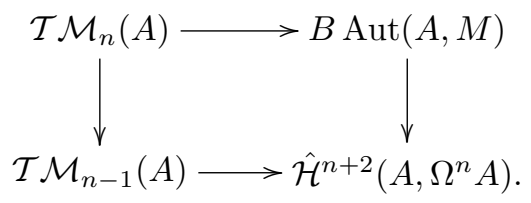

To interpret this result, let $Y \in \mathcal{T} \mathcal{M}_{n-1}(A)$ be a basepoint - that is, a potential $(n-1)$ st stage of of $A$. Then the homotopy fiber of $\mathcal{T} \mathcal{M}_{n}(A) \rightarrow$ $\mathcal{T} \mathcal{M}_{n-1}(A)$ is non-empty if and only if $Y$ is weakly equivalent to $P_{n-1} X$ for some potential $n$th stage of $A$. This, in turn, will occur if the image of $Y$ in $\pi_{0} \hat{\mathcal{H}}^{n+2}\left(A, \Omega^{n} A\right)$ is the zero element. Furthermore, if it is the zero element, then homotopy fiber at $Y$ is weakly equivalent to $\mathcal{H}^{n+1}\left(A, \Omega^{n} A\right)$. Therefore, by trying to lift the basepoint of $\mathcal{T} \mathcal{M}_{0}(A)=B \operatorname{Aut}(A)$ up the tower, we obtain the following corollary.

5.9 Corollary. There are successively defined obstructions, well defined up to the action of $\operatorname{Aut}(A, M)$,

$$
\theta_{n} \in D_{E_{*} T / E_{*} E}^{n+2}\left(A, \Omega^{n} A\right), \quad n \geq 1
$$

to realizing the $E_{*} \mathcal{F}$-algebra $A$ by an $\mathcal{F}$-algebra $X$. Obstructions to uniqueness lie in

$$
D_{E_{*} T / E_{*} E}^{n+1}\left(A, \Omega^{n} A\right) .
$$


Theorem 5.8 is proved exactly in the same manner as the main theorem of [6]. If one is interested only in the obstructions to realization, one can proceed as follows. Let $Y$ be a potential $(n-1)$ st stage for $A$. We'd like to construct a potential $n$th stage $X$ so that $P_{n-1} X \simeq Y$. We may assume that $Y$ is a cofibrant simplicial $T$-algebra. By a Postnikov section argument, we see that it is necessary and sufficient to produce a map of simplicial $T$-algebras over $B_{A}$

$$
Y \longrightarrow B_{A}\left(\Omega^{n} A, n+1\right)
$$

which induces an isomorphism on $\pi_{n+1}^{\natural}$. Because the space $B_{A}\left(\Omega^{n} A, n+1\right)$ represents André-Quillen cohomology, this is equivalent to producing a map of simplicial $E_{*} T$-algebras over $A$

$$
E_{*} Y \longrightarrow K_{A}\left(\Omega^{n} A, n+1\right)
$$

which (by calculating with the spiral exact sequence) is a weak equivalence. Since, as a simplicial $E_{*} T$-algebra, $E_{*} Y$ is a two-stage Postnikov tower, it is determined up to weak equivalence by a morphism in $s \operatorname{Alg}_{E_{*} T}$ over $A$

$$
A \simeq P_{0} E_{*} Y \longrightarrow K_{A}\left(\Omega^{n} A, n+2\right) .
$$

The class of this map in

$$
\pi_{0} \hat{\mathcal{H}}^{n+2}\left(A, \Omega^{n} A\right) \cong D_{E_{*} T / E_{*} E}^{n+2}\left(A, \Omega^{n} A\right) / \operatorname{Aut}\left(A, \Omega^{n} A\right)
$$

is the obstruction. The Borel construction is necessary as we have not fixed our various isomorphisms to $A$ and $\Omega^{n} A$.

The obstructions to uniqueness can found in Equation 4.9.

\section{Computing with $E_{\infty}$ operads.}

If Comm is the commutative monoid operad, then Theorem 2.1 supplies an augmented simplicial operad $T \rightarrow$ Comm so that the augmented simplicial operad $E_{*} T \rightarrow E_{*}$ Comm is an algebraic $E_{\infty}$ operad in a sense to be defined shortly. Since it is the simplicial operad $T$ and the methods of the previous section that we will use to attempt to impose $E_{\infty}$ structures on spectra, we need to be able to compute the André-Quillen cohomology functor $D_{E_{*} T}^{*}$. The purpose of this section is to reduce that computation, at least in some cases, to the calculation of ordinary André-Quillen homology or cohomology. The main result is the two spectral sequences supplied by the Propositions 6.4 and 6.5 below. Note that $E_{*}$ Comm is the commutative algebra operad in $E_{*}$ modules; hence ordinary André-Quillen cohomology is cohomology over the operad $E_{*}$ Comm.

We will first say what we mean by an $E_{\infty}$ operad. If $k$ is a commutative ring, we will write Comm for the commutative monoid operad in $k$-modules - rather than, for example, $k[\mathbf{C o m m}]$. 
6.1 Definition. For any commutative ring $k$ (possibly graded) an $E_{\infty}$-operad $\mathcal{E}$ is a simplicial operad in $k$-modules equipped with a weak equivalence $\mathcal{E} \rightarrow$ Comm and so that for each $q \geq 0, \mathcal{E}(q)$ is a cofibrant (i.e., level-wise projective) simplicial $k\left[\Sigma_{q}\right]$ module.

There is a canonical such operad - namely a cofibrant model for Comm in the category of simplicial $k$-operads - but we don't need that much structure in this discussion.

If $V_{*}$ is a cofibrant simplicial $k$-module, the shuffle chain equivalence of normalized chain complexes

$$
\stackrel{N(V) \otimes \cdots \otimes N(V)}{\longleftarrow} \rightarrow \stackrel{N(V \otimes \cdots \otimes V)}{\longleftarrow}
$$

is $\Sigma_{n}$-equivariant; thus if $C$ is any simplicial $k$-module operad, the normalized object $N C=\{N C(k)\}_{k \geq 0}$ is an operad of $k$-chain complexes. In particular, if $\mathcal{E}$ is an $E_{\infty}$ operad in the sense of Definition 6.1, then $N \mathcal{E}$ is an $E_{\infty}$ operad in the category of chain complexes over $k$. More is true. If $V$ is a simplicial $k$-module, and $\mathcal{C}$ is a simplicial operad, then there is a natural map of chain complexes

$$
N \mathcal{C}(q) \otimes_{k \Sigma_{q}} N V^{\otimes q} \rightarrow N\left(\mathcal{C}(q) \otimes_{k \Sigma_{q}} V^{\otimes q}\right)
$$

and if $\mathcal{C}(q)$ is cofibrant as a $k \Sigma_{q}$-module and $V$ is cofibrant as a $k$-module, this is a quasi-isomorphism. In shorthand,

$$
N \mathcal{C}(N V) \rightarrow N \mathcal{C}(V)
$$

is a quasi-isomorphism of $N \mathcal{C}$ algebras. Furthermore, if $A$ is any simplicial $\mathcal{C}$-algebra, $N A$ is an $N \mathcal{C}$ algebra via

$$
N \mathcal{C}(N A) \rightarrow N \mathcal{C}(A) \rightarrow N A .
$$

From these considerations, and from [28], it immediately follows that if $k$ is an algebra over a field $\mathbb{F}$ of characteristic $p>0$, and $\mathcal{E}$ is a simplicial $E_{\infty}$ operad, then the homotopy of any simplicial $\mathcal{E}$-algebra is an "unstable" algebra over the Dyer-Lashof algebra. That is, if $A \in s \operatorname{Alg}_{\mathcal{E}}$, then $\pi_{*} A$ is a graded commutative algebra equipped with operations

$$
Q^{i}: \pi_{n} A \rightarrow \pi_{n+i} A, \quad i \geq 0, p=2
$$

or

$$
\beta^{\epsilon} Q^{i}: \pi_{n} A \rightarrow \pi_{n+2 i(p-1)-\epsilon} A \quad i \geq 0, \epsilon=0,1, p>2
$$

subject to the Adem and Cartan formulas of [11], §I.1. Unstable in this context means, at $p=2$,

$$
Q^{i}(x)= \begin{cases}0 & i<\operatorname{deg}(x) \\ x^{2} & i=\operatorname{deg}(x)\end{cases}
$$

and at $p>2$

$$
\beta^{\epsilon} Q^{i}(x)= \begin{cases}0 & 2 i-\epsilon<\operatorname{deg}(x) \\ x^{p} & 2 i=\operatorname{deg}(x) \text { and } \epsilon=0 .\end{cases}
$$


This condition arises because we are dealing with a normalized object, not an arbitrary algebra over an $E_{\infty}$ operad in chain complexes.

Also if $k$ is not the prime field $\mathbb{F}_{p}$, these operations are not $k$-linear; if $\phi: k \rightarrow k$ is the Frobenius, $a \in k$ and $x \in \pi_{*} A$, then

$$
\beta^{\epsilon} Q^{i}(a x)=\phi(a) \beta^{\epsilon} Q^{i}(x) .
$$

There is an obvious category $\mathcal{U R}$ of unstable algebras over the Dyer-Lashof algebra. The forgetful functor $\mathcal{U R} \rightarrow n \mathcal{M}_{k}$ to graded $k$-modules has a left adjoint $\mathcal{S}_{\mathcal{R}}$. It follows from the quasi-isomorphisms of 6.1 and 6.2 and the calculations of [28] that if $\mathcal{E}$ is an $E_{\infty}$ operad and $V \in s \mathcal{M}_{k}$, then the natural map

$$
\mathcal{S}_{\mathcal{R}}\left(\pi_{*} V\right) \rightarrow \pi_{*} \mathcal{E}(V)
$$

is an isomorphism in $\mathcal{U} \mathcal{R}$ provided that $\pi_{*} V$ is a graded projective $k$-module. Note that this isomorphism does not depend on $\mathcal{E}$ : if $V$ a cofibrant simplicial $k$-module, $\pi_{*} \mathcal{E}(V)$ is independent of the $E_{\infty}$ operad $\mathcal{E}$ and we have:

6.2 Proposition. Let $f: \mathcal{E} \rightarrow \mathcal{E}^{\prime}$ be a morphism of $E_{\infty}$ operads over an $\mathbb{F}$ algebra $k$, where $\mathbb{F}$ is a field of positive characteristic. Then the restriction of structure functor and its left adjoint induce a Quillen equivalence

$$
\mathcal{E}^{\prime} \otimes_{\mathcal{E}}(\cdot)=f^{*}: s \operatorname{Alg}_{\mathcal{E}} \longleftrightarrow s \operatorname{Alg}_{\mathcal{E}^{\prime}}: f_{*} .
$$

This result is true over any ground ring $k$, although in general a less computational proof is required. Furthermore, any two $E_{\infty}$-operads are connected by a chain of such weak equivalences.

The algebra $S_{\mathcal{R}}(W)$ has a simple description, at least when $W$ is a graded projective $k$-module. See [11] §I.1. The operations $\beta^{\epsilon} Q^{i}$ can be assembled into an algebra $\mathcal{R}$ over $\mathbb{F}_{p}$ using the Adem relations (see [11], §I.2). This is the Dyer-Lashof algebra. If $W \in n \mathcal{M}_{k}$ is a graded $k$-module, let

$$
\mathcal{R}(W)=\mathcal{R} \otimes_{\mathbb{F}_{p}} W / U
$$

where $U$ is the sub- $\mathcal{R}$-module generated by elements of the form

$$
\begin{array}{ccc}
Q^{i} \otimes x, & i<\operatorname{deg}(x) & (p=2) \\
\beta^{\epsilon} Q^{i} \otimes x, & 2 i-\epsilon<\operatorname{deg}(x) & p>2 .
\end{array}
$$

Then, if $W$ is a graded projective $k$-module,

$$
S_{\mathcal{R}}(W)=S(\mathcal{R}(W)) / I
$$

where $S$ is the graded symmetric algebra functor over $k$ and $I$ is the ideal generated by the elements

$$
\begin{array}{lll}
Q^{i}(x)-x^{2} & \operatorname{deg}(x)=i, & p=2 \\
Q^{i}(x)-x^{p} & \operatorname{deg}(x)=2 i, & p>2
\end{array}
$$


In particular $S_{\mathcal{R}}(W)$ is a free graded commutative $k$-algebra.

If $\Gamma \in \mathcal{U R}$ then $\Gamma$ is, among other things, a graded commutative algebra and, as such, we can form its André-Quillen homology $D_{*} \Gamma$ as a graded commutative algebra:

$$
D_{*} \Gamma \stackrel{\text { def }}{=} \pi_{*} \mathbb{L}_{\Gamma / k}
$$

where $\mathbb{L}_{\Gamma / k}$ is the cotangent complex as a graded commutative algebra. As usual (cf. [29],[17]), the André-Quillen homology inherits structure from the Dyer-Lashof operations. We next spell out exactly what this structure is.

Let $\mathcal{U}$ be the category of non-negatively-graded modules over the DyerLashof algebras $\mathcal{R}$. These are graded $k$-modules and $\mathcal{R}$ acts with the Frobenius twist as in Equation 6.3, and unstable means that

$$
\begin{aligned}
& Q^{i}(x)=0 \quad \text { if } \quad i \leq \operatorname{deg}(x) \quad(p=2) \\
& \beta^{\epsilon} Q^{i}(x)=0 \quad \text { if } \quad 2 i-\epsilon<\operatorname{deg}(x) \text { or } 2 i=\operatorname{deg}(x) \quad(p>2)
\end{aligned}
$$

If $\Gamma \in \mathcal{U R}$ then $\mathcal{U}(\Gamma)$ is the category of objects $M$ which are at once in $\mathcal{U}$, and graded $\Gamma$-modules subject to the compatibility condition that the multiplication map

$$
\Gamma \otimes_{k} M \rightarrow M
$$

is a morphism in $\mathcal{U}$.

Such structures arise naturally as follows: If $M \in \mathcal{U}(\Gamma)$, let $\operatorname{Der}_{\mathcal{R}}(\Gamma, M)$ be the module of commutative $k$-algebra derivations that commute with the elements of $\mathcal{R}$. The following is proved with a minor variation of the (entirely standard) techniques of [17] $§ 1$.

6.3 Lemma. Let $\Gamma \in \mathcal{U} \mathcal{R}$. The graded module $\Omega_{\Gamma / k}$ of commutative algebra derivations is naturally an object in $\mathcal{U}(\Gamma)$ and there is a natural isomorphism

$$
\operatorname{Der}_{\mathcal{R}}(\Gamma, M) \cong \operatorname{Hom}_{\mathcal{U}(\Gamma)}\left(\Omega_{\Gamma / k}, M\right) .
$$

The functor which assigns to an algebra $\Gamma \in \mathcal{U} \mathcal{R}$ the module of derivations $\operatorname{Der}_{\mathcal{R}}(\Gamma, M)$ has non-abelian right derived functors. Choose a cofibrant $X$ model for $\Gamma \in \mathcal{U} \mathcal{R}$ regarded as a constant object in $s \mathcal{U R}$. Then these derived functors are a kind of André-Quillen cohomology:

$$
D_{\mathcal{R}}^{n}(\Gamma, M)=\pi^{n} \operatorname{Der}_{\mathcal{R}}(X, M) .
$$

This cohomology can be dissected. We may assume $X_{q}=S_{\mathcal{R}}\left(V_{q}\right)$ for some graded, projective $k$-module $V_{q}$; hence, as a simplicial graded commutative algebra, $X \rightarrow \Gamma$ is still a cofibrant model for $\Gamma$. Thus

$$
\mathbb{L}_{\Gamma / k} \simeq \Gamma \otimes_{X} \Omega_{X / k}
$$

acquires, by Lemma 6.3, the structure of a cofibrant simplicial $s \mathcal{U}(\Gamma)$ module. This implies that the ordinary André-Quillen homology

$$
D_{*} \Gamma \cong \pi_{*} \mathbb{L} \Omega_{\Gamma / k}
$$


is a graded object in $\mathcal{U}(\Gamma)$, and this structure is independent of the choice of $X$.

This noted, it is not surprising that the natural isomorphism of Lemma 6.3 yields a composite functor spectral sequence:

6.4 Proposition. Let $\Gamma \in \mathcal{U} \mathcal{R}$. Then there is a spectral sequence

$$
\operatorname{Ext}_{\mathcal{U}(\Gamma)}^{p}\left(D_{q}(\Gamma), M\right) \Rightarrow D_{\mathcal{R}}^{p+q}(\Gamma, M) .
$$

This is important because of the following result. Let $k$ be an algebra over a field of positive characteristic, and $\mathcal{E}$ a simplicial $E_{\infty}$ operad over $k$. If $A$ is a simplicial $\mathcal{E}$ algebra and $M$ is a $\pi_{0} A$ module (over the operad $\pi_{0} \mathcal{E}=\mathbf{C o m m}$ ), then $M$ is an object in $\mathcal{U}\left(\pi_{*} A\right)$.

6.5 Proposition. Let $\mathcal{E}$ be an $E_{\infty}$ operad over an $\mathbb{F}$-algebra $k$, where $\mathbb{F}$ is a field of characteristic $p>0$. Let $A \in s \operatorname{Alg}_{\mathcal{E}}$. Then there is a spectral sequence

$$
D_{\mathcal{R}}^{p}\left(\pi_{*} A, M\right)^{q} \Rightarrow D_{\mathcal{E}}^{p+q}(A, M) .
$$

Proof. Here is an outline of the proof. We may assume $A$ is cofibrant. Let $P_{\bullet}^{\mathcal{E}} A \in s\left(s \mathcal{A}^{T}\right)$ be a simplicial resolution of $A$ by $\mathcal{E}$ algebras of the form $\mathcal{E}(W)$ where $W$ is a cofibrant simplicial $k$-module with the property that $\pi_{*} W$ is a projective $k$-module. Here resolution means that

$$
\pi_{*} \pi_{*} P_{\bullet}^{\mathcal{E}} A \cong \pi_{*} A
$$

via the augmentation. It is possible to construct such by a Stover resolution argument. Compare section 3. Note that this and Equation 6.4 imply that

$$
\pi_{*} P_{\bullet}^{\mathcal{E}} A \longrightarrow \pi_{*} A
$$

is a cofibrant model for $\pi_{*} A$ as a simplicial object in $\mathcal{U} \mathcal{R}$.

Taking the geometric realization, which is possible because $s \operatorname{Alg}_{\mathcal{E}}$ is a simplicial model category, we obtain a weak equivalence

$$
\left|P_{\bullet}^{\mathcal{E}} A\right| \longrightarrow A
$$

and hence a spectral sequence

$$
\pi^{p} D_{\mathcal{E}}^{q}\left(P_{\bullet}^{\mathcal{E}}(A), M\right) \Longrightarrow D^{p+q}\left(\left|P_{\bullet}^{\mathcal{E}} A\right|, M\right) \cong D^{p+q}(A, M)
$$

The last isomorphism follows because $\left|P_{\bullet}^{\mathcal{E}} A\right|$ is cofibrant. The claim is that

$$
D_{\mathcal{E}}^{*}(\mathcal{E}(W), M) \cong \operatorname{Der}_{\mathcal{R}}\left(S_{\mathcal{R}}\left(\pi_{*} W\right), M\right) .
$$

This is easily verified, completing the proof.

6.6 Remark. If $k$ is an algebra over a field of characteristic 0 and $\mathcal{E}$ is an $E_{\infty}$-operad for $k$-modules, then the weak equivalence of simplicial operads $\epsilon$ : $\mathcal{E} \rightarrow$ Comm induces a Quillen equivalence

$$
\epsilon^{*}: s \operatorname{Alg}_{\mathcal{E}} \rightleftarrows s \operatorname{Alg}_{\text {Comm }}: \epsilon_{*} .
$$

Furthermore, André-Quillen cohomology over $\mathcal{E}$ reduces to André-Quillen cohomology for commutative $k$-algebras. 
In our applications, we will encounter simplicial algebras of the form $E_{*} X$ where $X$ is some simplicial algebra over some simplicial operad. In this case, the ground ring will be $k=E_{*}$ and very rarely will this be an algebra over a field of characteristic $p$. Therefore, we close this section with two results intended to reduce calculations to the case considered above.

The first is this. Suppose $\mathfrak{m} \subseteq k$ is an ideal with the property that $k / \mathfrak{m}$ is an algebra over a field of chacteristic $p$. Then if $\mathcal{E}$ is an $E_{\infty}$ operad over $k$ in the sense of Definition 6.1, then $k / \mathfrak{m} \otimes_{k} \mathcal{E}$ is an $E_{\infty}$ operad over $k / \mathfrak{m}$. Furthermore, if $A$ is a simplicial $\mathcal{E}$ algebra, then $k / \mathfrak{m} \otimes_{k} A$ is a simplicial $k / \mathfrak{m} \otimes_{k} \mathcal{E}$ algebra. If $M$ is module over

$$
\pi_{0}\left(k / \mathfrak{m} \otimes_{k} A\right) \cong k / \mathfrak{m} \otimes_{k} \pi_{0} A
$$

then $M$ is a module over $\pi_{0} A$ and we'd like to use these facts to compute $D_{\mathcal{E}}^{*}(A, M)$. If $X$ is a cofibrant $\mathcal{E}$ algebra, then $X$ is cofibrant as simplicial $k$ module; hence if $X \rightarrow A$ is a weak equivalence of $\mathcal{E}$ algebras with $X$ cofibrant, then $k / \mathfrak{m} \otimes_{k} X$ is a model for the derived tensor product $k / \mathfrak{m} \otimes_{k}^{\mathbb{L}} A$.

6.7 Proposition. Let $A$ be a simplicial $\mathcal{E}$ algebra over an $E_{\infty}$ operad over $k$ and let $M$ be a $k / \mathfrak{m} \otimes_{k} \pi_{0} A$ module. Then there is a natural isomorphism

$$
D_{\mathcal{E}}^{*}(A, M) \cong D_{k / \mathfrak{m} \otimes_{k} \mathcal{E}}^{*}\left(k / m \otimes_{k}^{\mathbb{L}} A, M\right) .
$$

If $\pi_{*} A$ is flat over $k$, then

$$
\pi_{*}\left(k / \mathfrak{m} \otimes_{k}^{\mathbb{L}} A\right) \cong k / \mathfrak{m} \otimes_{k} \pi_{*} A .
$$

Now suppose $M$ is simply a module over $\pi_{0} A$ and suppose that $M$ is flat as a $k$-module. Then we can filter the module $M$ by powers of the ideal $\mathfrak{m} \subseteq k$ to get a spectral sequence:

6.8 Proposition. There is a spectral sequence

$$
E_{1}^{p, q}=D_{k / \mathfrak{m} \otimes_{k} \mathcal{E}}^{p}\left(k / \mathfrak{m} \otimes_{k}^{\mathbb{L}} A, \mathfrak{m}^{q} M / \mathfrak{m}^{q+1} M\right) \Longrightarrow \lim _{q} D_{\mathcal{E}}^{p}\left(A, M / \mathfrak{m}^{q} M\right) .
$$

If $M$ is $\mathfrak{m}$-complete in the sense that $M \cong \lim _{q} M / \mathfrak{m}^{q} M$ and there is an $r$ so that for all $(p, q)$ we have $E_{r}^{p, q}=E_{\infty}^{p, q}$, then

$$
\lim _{q} D_{\mathcal{E}}^{p}\left(A, M / \mathfrak{m}^{q} M\right) \cong D_{\mathcal{E}}^{q}(A, M)
$$

\section{The Lubin-Tate Theories}

In the section we apply the technology developed in the previous sections to show that the techniques used by Haynes Miller and the second author (cf. [34]) to show that the algebraic theory of deformations of height $n$ formal group laws actually lifts to $E_{\infty}$-ring spectra.

The Lubin-Tate theory [25] of deformations of finite height formal group laws works over an arbitrary perfect field of characteristic $p$. However, we will 
specialize to algebraic extensions of the prime field $\mathbb{F}_{p}$ to keep the language simple.

Fix a such a field $k$ and a formal group law $\Gamma$ over $k$. A deformation of $\Gamma$ to a complete local ring $A$ (with maximal ideal $\mathfrak{m}$ ) is a pair $(G, i)$ where $G$ is a formal group law over $A, i: k \rightarrow A / \mathfrak{m}$ is a morphism of fields and one requires $i^{*} \Gamma=\pi^{*} G$, where $\pi: A \rightarrow A / \mathfrak{m}$ is the quotient map. Two such deformations $(G, i)$ and $(H, j)$ are $\star$-isomorphic if there is an isomorphism $f: G \rightarrow H$ of formal group laws which reduces to the identity modulo $\mathfrak{m}$. Write $\operatorname{Def}_{\Gamma}(A)$ for the set of $\star$-isomorphism classes of deformations of $\Gamma$ over $A$.

A common abuse of notation is to write $G$ for the deformation $(G, i) ; i$ is to be understood from the context.

Now suppose the height of $\Gamma$ is finite. Then the theorem of Lubin and Tate [25] says that the functor $A \mapsto \operatorname{Def}_{\Gamma}(A)$ is representable. Indeed let

$$
A(\Gamma, k)=W(k)\left[\left[u_{1}, \cdots, u_{n-1}\right]\right]
$$

where $W(k)$ denotes the Witt vectors on $k$ and $n$ is the height of $\Gamma$. This is a complete local ring with maximal ideal $\mathfrak{m}=\left(p, u_{1}, \cdots, u_{n-1}\right)$ and there is a canonical isomorphism $q: k \cong A(\Gamma, k) / \mathfrak{m}$. Then Lubin and Tate prove there is a deformation $(G, q)$ of $\Gamma$ over $A(\Gamma, k)$ so that the natural map

$$
\operatorname{Hom}_{c}(A(\Gamma, k), A) \rightarrow \operatorname{Def}_{\Gamma}(A)
$$

sending a continuous map $f: A(\Gamma, k) \rightarrow A$ to $\left(f_{*} G, \bar{f} q\right)$ (where $\bar{f}$ is the map on residue fields induced by $f$ ) is an isomorphism. Continuous maps here are very simple: they are the local maps; that is, we need only require that $f(\mathfrak{m})$ be contained in the maximal ideal of $A$. Furthermore, if two deformations are $\star$-isomorphic, then the $\star$-isomorphism between them is unique.

We'd like to now turn the assignment $(\Gamma, k) \mapsto A(\Gamma, k)$ into a functor. For this we introduce the category $\mathcal{F} \mathcal{G} \mathcal{L}_{n}$ of height $n$ formal group laws over fields which are algebraic extensions of $\mathbb{F}_{p}$. The objects are pairs $(\Gamma, k)$ where $\Gamma$ is of height $n$. A morphism

$$
(f, j):\left(\Gamma_{1}, k_{1}\right) \rightarrow\left(\Gamma_{2}, k_{2}\right)
$$

is a homomorphism of fields $j: k_{1} \rightarrow k_{2}$ and an isomorphism of formal group laws $f: j^{*} \Gamma_{1} \rightarrow \Gamma_{2}$. This is the opposite of the category considered by Rezk in [34]. We make this choice so we get a covariant functor. As a result, some of our results below also have an opposite flavor - nonetheless, these are the same results.

Let $(f, j)$ be such a morphism and let $G_{1}$ and $G_{2}$ be the fixed universal deformations over $A\left(\Gamma_{1}, k\right)$ and $A\left(\Gamma_{2}, k\right)$ respectively. If $\bar{f} \in A\left(\Gamma_{2}, k_{2}\right)[[x]]$ is any lift of $f \in k_{2}[[x]]$, then we can define a formal group law $H$ over $A\left(\Gamma_{2}, k_{2}\right)$ by requiring that $\vec{f}: H \rightarrow G_{2}$ is an isomorphism. Then the pair $(H, j)$ is a deformation of $\Gamma_{1}$, hence we get a homomorphism $A\left(\Gamma_{1}, k_{1}\right) \rightarrow A\left(\Gamma_{2}, k_{2}\right)$ classifying the $\star$-isomorphism class of $H$-which, one easily checks, is independent 
of the lift $\bar{f}$. Thus if Rings $s_{c}$ is the category of complete local rings and local homomorphims, we get a functor

$$
A(\cdot, \cdot): \mathcal{F} \mathcal{G L}_{n} \longrightarrow \text { Rings }_{c} .
$$

In particular, note that any morphism in $\mathcal{F} \mathcal{G} \mathcal{L}_{n}$ from a pair $(\Gamma, k)$ to itself is an isomorphism. Thus, these endomorphisms form the "big" Morava stabilizer group of the formal group law. It contains the usual Morava stabilizer group as the subgroup of elements of the form $\left(f, i d_{k}\right)$. The formal group law and hence also its automorphism group is determined up to isomorphism by the height of $\Gamma$ if $k$ is separably closed.

Next we put in the gradings. This requires a paragraph of introduction. For any commutative ring $R$, the morphism $R[[x]] \rightarrow R$ of rings sending $x$ to 0 makes $R$ into a $R[[x]]$-module. Let $\operatorname{Der}_{R}(R[[x]], R)$ denote the $R$-module of continuous $R$-derivations; that is, continuous $R$-module homomorphisms

$$
\partial: R[[x]] \longrightarrow R
$$

so that

$$
\partial(f(x) g(x))=\partial(f(x)) g(0)+f(0) \partial(g(x)) .
$$

If $\partial$ is any derivation, write $\partial(x)=u$; then, if $f(x)=\sum a_{i} x^{i}$,

$$
\partial(f(x))=a_{1} \partial(x)=a_{1} u .
$$

Thus $\partial$ is determined by $u$, and we write $\partial=\partial_{u}$. We then have that the module $\operatorname{Der}_{R}(R[[x]], R)$ is a free $R$-module of rank one, generated by any derivation $\partial_{u}$ so that $u$ is a unit in $R$. In the language of schemes, $\partial_{u}$ is a generator for the tangent space at 0 of the formal scheme $\mathbb{A}_{R}^{1}$ over $\operatorname{Spec}(R)$.

Now consider pairs $(F, u)$ where $F$ is a formal group law over $R$ and $u$ is a unit in $R$. Thus $F$ defines a smooth one dimensional commutative formal group scheme over $\operatorname{Spec}(R)$ and $\partial_{u}$ is a chosen generator for the tangent space at 0 . A morphism of pairs

$$
f:(F, u) \longrightarrow(G, v)
$$

is an isomorphism of formal group laws $f: F \rightarrow G$ so that

$$
u=f^{\prime}(0) v .
$$

Note that if $f(x) \in R[[x]]$ is a homomorphism of formal group laws from $F$ to $G$, and $\partial$ is a derivation at 0 , then $\left(f^{*} \partial\right)(x)=f^{\prime}(0) \partial(x)$. In the context of deformations, we may require that $f$ be a $\star$-isomorphism.

This suggests the following definition: let $\Gamma$ be a formal group law of height $n$ over a field $k$ which is an algebraic extension of $\mathbb{F}_{p}$ and let $A$ be a complete local ring. Define $\operatorname{Def}_{\Gamma}(A)_{*}$ to be equivalence classes of pairs $((G, i), u)$ where $(G, i)$ is a deformation of $\Gamma$ to $A$ and $u$ is a unit in $A$. The equivalence relation is given by $\star$-isomorphisms transforming the unit as in the last paragraph. We now have that there is a natural isomorphism

$$
\operatorname{Hom}_{c}\left(A(\Gamma, k)\left[u^{ \pm 1}\right], A\right) \cong \operatorname{Def}_{\Gamma}(A)_{*} .
$$


We impose a grading by giving an action of the multiplicative group scheme $\mathbb{G}_{m}$ on the scheme $\operatorname{Def}_{\Gamma}(\cdot)_{*}$ (on the right) and thus on $A(\Gamma, k)\left[u^{ \pm 1}\right]$ (on the left): if $v \in A^{\times}$is a unit and $(G, u)$ represents an equivalence class in $\operatorname{Def}_{\Gamma}(A)_{*}$ define an new element in $\operatorname{Def}_{\Gamma}(A)_{*}$ by $\left(G, v^{-1} u\right)$. In the induced grading on $A(\Gamma, k)\left[u^{ \pm 1}\right]$, one has $A(\Gamma, k)$ in degree 0 and $u$ in degree -2 .

This grading is essentially forced by topological considerations. See the remarks before Theorem 20 of [39] for an explanation.

We now collect a sequence of results, mostly from Rezk's paper [34], to develop the input to our machine.

7.1 Proposition. For all pairs $(\Gamma, k) \in \mathcal{F} \mathcal{G} \mathcal{L}_{n}$, the universal deformation over $A(\Gamma, k)\left[u^{ \pm 1}\right]$ is a Landweber exact formal group law. Furthermore, the resulting homology theory $E(\Gamma, k)_{*}$ is of Adams-type.

Proof. See Propositions 6.5 and 15.3 of [34].

We will write $E(\Gamma, k)$ for the representing spectrum of this homology theory.

7.2 Remark. The importance of these homology theories - and of the whole moduli problem we are discussing here - was first recognized by Morava. Hence we might call these homology theories Morava $E$-theories. If we choose $k=\mathbb{F}_{p^{n}}$ and $\Gamma$ to be the Honda formal group law of height $n$, the $E(\Gamma, k)_{*}$ is what is commonly written $\left(E_{n}\right)_{*}$. A mild variant of the resulting spectrum was shown to be an $A_{\infty}$-ring spectrum by Baker [3]; his methods apply equally to all of the spectra $E(k, \Gamma)$.

Note that the ring $E(\Gamma, k)_{0} \cong A(\Gamma, k)$ and, hence, it is a complete local ring. Fix two objects $\left(\Gamma_{1}, k_{1}\right)$ and $\left(\Gamma_{2}, k_{2}\right)$ is $\mathcal{F} \mathcal{G} \mathcal{L}_{n}$ and let $F=E\left(\Gamma_{1}, k_{1}\right)$, $E=E\left(\Gamma_{2}, k_{2}\right)$

7.3 Proposition. Let $A_{*}$ be a graded commutative ring so that $A_{0}$ is a complete local ring with maximal ideal $\mathfrak{m}$. Suppose $i: E_{*} \rightarrow A_{*}$ is a morphism of graded commutative rings which is continuous in degree 0 . Then the set

$$
\operatorname{Hom}_{E_{*}-a l g}\left(E_{*} F, A_{*}\right)
$$

is isomorphic to the set of morphisms in $\mathcal{F} \mathcal{G} \mathcal{L}_{n}$

$$
\left(\Gamma_{1}, k_{1}\right) \rightarrow\left(i^{*} \Gamma_{2}, A_{0} / \mathfrak{m}\right)
$$

Proof. This is a consequence of Landweber exactness and the groupoid point of view to deformations. See section $\S 17$ of [34].

For example, if we set $A_{*}=E_{*}$, the get that

$$
\operatorname{Hom}_{E_{*}-\operatorname{alg}}\left(E_{*} F, E_{*}\right)=\operatorname{Hom}_{\mathcal{F} \mathcal{G} \mathcal{L}_{n}}\left(\left(\Gamma_{1}, k_{1}\right),\left(\Gamma_{2}, k_{2}\right)\right) .
$$

If $k$ is field of characteristic $p$ and $A$ a $k$-algebra, let $\sigma: A \rightarrow A$ denote the Frobenius. This is not a $k$-algebra homomorphism, but the commutative 
diagram

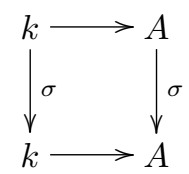

yields a $k$-algebra homomorphism

$$
\sigma: k \otimes_{\sigma} A \rightarrow A
$$

called the relative Frobenius. Now let $\mathfrak{m}_{E} \subseteq E_{0} F$ be extension of the maximal ideal $\mathfrak{m} \subseteq A\left(\Gamma_{1}, k_{1}\right)=E_{0}$; that is $\mathfrak{m}_{E}=\mathfrak{m} E_{0} F$ and

$$
E_{0} F / \mathfrak{m}_{E}=k_{1} \otimes_{E_{0}} E_{0} F
$$

7.4 Proposition. The relative Frobenius

$$
\sigma: k_{1} \otimes_{\sigma} E_{0} F / \mathfrak{m}_{E} \rightarrow E_{0} F / \mathfrak{m}_{E}
$$

is an isomorphism. As a consequence

$$
\mathbb{L}_{k_{1} \otimes_{E_{0}} E_{0} F / k_{1}} \simeq 0
$$

Proof. The first statement follows easily from Proposition 7.3 and facts about powers series. See [34], Proposition 21.5. The second statement follows from the fact that

$$
\left.\sigma_{*}: \mathbb{L}_{\left(k_{1} \otimes R\right.} E_{0} F\right) / k_{1} \rightarrow \mathbb{L}_{E_{0} F / k_{1}}
$$

is both an isomorphism and the zero map. See Proposition 21.2 of [34].

7.5 Corollary. The graded cotangent complex is contractible:

$$
\mathbb{L}_{\left(k_{1} \otimes_{E_{0}} E_{*} F\right) /\left(k_{1}\left[u^{ \pm 1}\right)\right]} \simeq 0 .
$$

Proof. This is a consequence of the previous result and flat base-change (see [30]) for the square

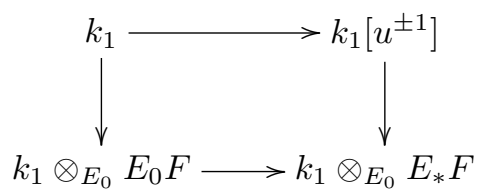

7.6 Corollary. The moduli space of a realizations of $E(\Gamma, k)_{*} E(\Gamma, k)$ as a commutative $E(\Gamma, k)_{*}$ algebra in $E(\Gamma, k)_{*} E(\Gamma, k)$-comodules has the homotopy type of

$$
B \operatorname{Aut}(\Gamma, k)
$$

where the automorphism group is computed in $\mathcal{F} \mathcal{G} \mathcal{L}_{n}$. In particular, $E(\Gamma, k)$ has a unigue $E_{\infty}$-structure realizing $E(\Gamma, k)_{*} E(\Gamma, k)$ as a commutative $E(\Gamma, k)_{*}$ algebra. 
Proof. Let's write $E_{*}$ and $E_{*} E$ for $E(\Gamma, k)_{*}$, etc. We first show $\mathcal{T} \mathcal{M}\left(E_{*} E\right) \simeq$ $B \operatorname{Aut}\left(E_{*} E\right)$. Putting together the decomposition of the moduli space given Proposition 5.2, Proposition 5.5, and Theorem 5.8, we see that it is sufficient to calculate that

$$
D_{E_{*} T / E_{*} E}\left(E_{*} E, \Omega^{t} E_{*} E\right)=0
$$

for all $t$. By Proposition 4.2, these groups are isomorphic to

$$
D_{E_{*} T}^{*}\left(E_{*} E, \Omega^{t} E_{*}\right) .
$$

Now Proposition 6.8, and the spectral sequences of Propositions 6.4 and 6.5, and the previous result imply that this latter cohomology group is zero.

To finish the result we see that Proposition 7.3 - or more exactly its consequence Equation 7.3 - implies that

$$
\operatorname{Aut}\left(E_{*} E\right) \cong \operatorname{Aut}(\Gamma, k) .
$$

7.7 Corollary. Let $E\left(\Gamma_{i}, k_{i}\right)$ be two of the Lubin-Tate $E_{\infty}$ ring spectra. Then the space of $E_{\infty}$-maps between these spectra has contractible components; furthemore the set of path components is isomorpic to the set of morphisms

$$
\left(\Gamma_{1}, k_{1}\right) \rightarrow\left(\Gamma_{2}, k_{2}\right)
$$

is $\mathcal{F} \mathcal{G} \mathcal{L}_{n}$.

Proof. This is the same line of argument, where the mapping space is decomposed via the spectral sequence of Theorem 4.3 or 4.5 .

\section{References}

[1] Adams, J.F., Stable homotopy and generalised cohomology, University of Chicago Press, Chicago, 1974.

[2] Atiyah, M. F., "Vector bundles and the Künneth formula", Topology, 1 (1962), 245-248.

[3] Baker, A., " $A_{\infty}$ structures on some spectra related to Morava $K$-theories", Quart. J. Math. Oxford Ser. (2), 42 (1991), No. 168, 403-419.

[4] Basterra, M., "André-Quillen cohomology of commutative $S$-algebras", $J$. Pure and Applied Algebra, to appear.

[5] Berger, C. and Moerdijk, I," "Axiomatic homotopy theory for operads", preprint 2002,

http://front.math.ucdavis. edu/math. AT/0206094. 
[6] Blanc, D., Dwyer, W., and Goerss, P., "The realization space of a Пalgebra: a moduli problem in algebraic topology", preprint 2002, available at the Hopf archive.

[7] Bousfield, A. K., "Homotopy spectral sequences and obstructions", Isr. J. Math. 66 (1989) 54-104.

[8] Bousfield, A. K. and Kan, D. M., Homotopy limits, completions, and localizations, Lecture Notes in Math. 304 ( $2^{\text {nd }}$ corrected printing), SpringerVerlag, Berlin-Heidelberg-New York, 1987.

[9] Bousfield, A. K., "Cosimplicial resolutions and homotopy spectral sequences in model categories", manuscript, University of Illinois at Chicago, 2001.

[10] Bousfield, A. K. and Friedlander, E. M., "Homotopy theory of $\Gamma$-spaces, spectra, and bisimplicial sets", Geometric applications of homotopy theory (Proc. Conf., Evanston, Ill., 1977), II, Lecture Notes in Math, 658, 80-130, Springer-Verlag, Berlin 1978.

[11] Cohen, F. R., Lada, T., and May, J. P., The Homology of Iterated Loop Spaces, Lecture Notes in Mathematics, Vol. 533, Springer-Verlag, Berlin, 1976.

[12] Dwyer, W. G. and Kan, D. M., "A classification theorem for diagrams of simplicial sets", Topology, 23 (1984) No.2, 139-155.

[13] Dwyer, W. G. and Kan, D. M., "Function complexes in homotopical algebra, Topology, 18 (1980), No. 4, 427-440.

[14] W. G. Dwyer, D. M. Kan, C. R. Stover, $E_{2}$ model category structure for pointed simplicial spaces," J. of Pure and Applied Algebra 90 (1993), 137152.

[15] W. G. Dwyer, D. M. Kan, C. R. Stover, "The bigraded homotopy groups $\pi_{i, j} X$ of a pointed simplicial space X", J. of Pure and Applied Algebra 103 (1995), 167-188.

[16] A. D. Elmendorff, I. Kriz, M. A. Mandell, J. P. May, "Rings, modules, and algebras in stable homotopy theory", Mathematical Surveys and Monographs 47, AMS, Providence, RI, 1996.

[17] Goerss, P. G., "André-Quillen cohomology and the homotopy groups of mapping spaces: understanding the $E_{2}$ term of the Bousfield-Kan spectral sequence," J. of Pure and Applied Algebra 63 (1990), pp. 113-153.

[18] Goerss, P. G. and Hopkins, M. J., "André-Quillen (co)-homology for simplicial algebras over simplicial operads", Une dégustation topologique: homotopy theory in the Swiss Alps (Arolla, 1999), Contemp. Math., 265, 41-85, Amer. Math. Soc., Providence,RI, 2000. 
[19] Heller, A., "On the representability of homotopy functors", J. London Math. Soc. 23 (1981), 551-562.

[20] Hirschhorn, P., Model categories and their localizations, Mathematical Surveys and Monographs, 99, American Mathematical Society, Providence, RI, 2002.

[21] Hovey, M., Model categories, Mathematical Surveys and Monographs, 63, American Mathematical Society, Providence, RI, 1999.

[22] Hovey, M. and Shipley, B. and Smith, J., "Symmetric spectrai", J. Amer. Math. Soc., 13 (2000) No. 1, 149-208.

[23] Illusie, L, Complexe cotangent et déformations. I, Lecture Notes in Mathematics, Vol. 239, Springer-Verlag, Berlin, 1971.

[24] Jardine, J. F., "Bousfield's $E_{2}$ model theory for simplicial objecst", preprint University of Western Ontario, 2002.

[25] Lubin, J. and Tate, J., "Formal moduli for one-parameter formal Lie groups", Bull. Soc. Math. France 94 (1966), 49-60.

[26] Lewis, L. G., Jr., May, J. P., Steinberger, M., Equivariant Stable Homotopy Theory, Lecture Notes in Mathematics 1213, Springer-Verlag, Berlin, 1986.

[27] Mandell, M. A. and May, J. P. and Schwede, S. and Shipley, B., "Model categories of diagram spectra", Proc. London Math. Soc. (3), 82 (2001), No. 2, 441-512.

[28] May, J.P., "A general approach to Steenrod operations", The Steenrod Algebra and its Applications (Proc. Conf. to Celebrate N.E. Steenrod's Sixtieth Birthday, Battelle Memorial Inst. Columbus, Ohio, 1970, pp. 153-231, Lecture Notes in Mathematics, Vol. 168, Springer-Verlag, Berlin.

[29] Miller, H., "The Sullivan conjecture on maps from classifying spaces," Ann. of Math. 120 (1984), pp. 39-87.

[30] Quillen, D.G., On the (co)-homology of commutative rings, Proc. Symp. Pure Math. 17 (1970), 65-87.

[31] Quillen D.G., Homotopical Algebra, Lecture Notes in Math. 43, SpringerVerlag, Berlin-Heidelberg-New York, 1967.

[32] Reedy, C. L., "Homotopy theory of model categories", Preprint, 1973. Available from http://math.mit.edu/ ${ }^{\sim}$ psh.

[33] Rezk, C. W., "Spaces of algebra structures and cohomology of operads", Thesis, MIT, 1996.

[34] Rezk, C. W., "Notes on the Hopkins-Miller theorem", in Homotopy Theory via Algebraic Geometry and Group Representations, M. Mahowald and S. Priddy, eds., Contemporary Math. 220 (1998) 313-366. 
[35] Robinson, A., "Obstruction theory and the strict associativity of Morava K-theory," Advances in homotopy theory, London Math. Soc. Lecture Notes 139 (1989), 143-152.

[36] Robinson, A., "Gamma homology, Lie representations and $E_{\infty}$ multiplications", Invent. Math., 152 (2003) No. 2, 331-348.

[37] Robinson, A. and Whitehouse, S., "Operads and $\Gamma$-homology of commutative rings", Math. Proc. Cambridge Philos. Soc., 132 (2002), No. 2, 197-234.

[38] Stover, C. R., "A Van Kampen spectral sequence for higher homotopy groups," Topology 29 (1990), 9-26.

[39] Strickland, N. P., "Gross-Hopkins duality", Topology, 39 (2000) No. 5 (1021-1033).

Department of Mathematics, Northwestern University, Evanston IL 60208 pgoerss@math.nwu.edu

Department of Mathematics, MIT, Cambridge MA, 02139

mjh@math.mit.edu 